\title{
Emergent 2HDM in the Low-Skiba-Smith little Higgs model: Musings from flavor and electroweak physics
}

\author{
Nilanjana Kumar® $\circledast^{1, *}$ and Soumya Sadhukhan ${ }^{1,2, \dagger}$ \\ ${ }^{1}$ Department of Physics and Astrophysics, University of Delhi, New Delhi, 110007, India \\ ${ }^{2}$ Ramakrishna Mission Residential College (Autonomous), Narendrapur, Kolkata 700103, India
}

(Received 21 August 2020; accepted 8 February 2021; published 15 March 2021)

\begin{abstract}
The low-energy effective theory ( $\sim \mathrm{TeV})$ of the little Higgs model with $S U(6) / S p(6)$, as proposed by Low, Skiba, and Smith (LSS), exhibits a two-Higgs-doublet model (2HDM) structure. The symmetry dictates interesting Yukawa patterns, translating to nontrivial fermion couplings with both of the Higgs doublets. The couplings of the scalars with the fermions can induce flavor-changing neutral currents (FCNC), which get constraints from flavor physics observables such as $\operatorname{BR}\left(B \rightarrow X_{s} \gamma\right), B_{s}-\bar{B}_{s}$ mixing, etc. The precision measurement of the $Z b \bar{b}$ vertex, the top and Higgs masses along with other Higgs coupling measurements at the Large Hadron Collider (LHC) also enforce severe restrictions on the LSS model. Direct LHC search results of beyond-the-Standard-Model (BSM) particles also impose bounds on the masses. We probe the LSS model in view of the above constraints through a random scan in the multidimensional parameter space. We observe, contrary to the general 2HDM scenario, that the emergent 2HDM from the LSS model is less constrained from the flavor data and the $Z b \bar{b}$ measurement but is severely constrained from the electroweak (EW) searches at the LHC. From the flavor data and $Z b \bar{b}$, we find that the charged Higgs mass is relaxed, with $\tan \beta$ being restricted to $0.5-5$, whereas the charged Higgs mass is pushed to larger than $1 \mathrm{TeV}$ along with $\tan \beta$ being further restricted to $<3$ when the LHC bounds are incorporated.
\end{abstract}

DOI: 10.1103/PhysRevD.103.055011

\section{INTRODUCTION}

Even if the Standard Model (SM) is the favorite candidate to explain all the results obtained at the Large Hadron Collider (LHC), models with extended symmetries beyond the SM are of great interest due to their elegant UV completion along with the power of stabilizing the scalars against radiative corrections-i.e., solving the gauge hierarchy problem [1]. There are different broad classes of models with enhanced symmetries. First, there are models where the space-time symmetry is enhanced to incorporate the spin, and also to have symmetries that even relate the bosons and the fermions; these are called supersymmetric models [2]. Another set of models also possess enhanced gauge symmetries which are systematically broken, sometimes in multiple steps, in order for the Higgs to emerge as a pseudo-Nambu-Goldstone boson (pNGB). In these models, such as the little Higgs [3] and composite Higgs [4]

*nilanjana.kumar@gmail.com

†physicsoumya@gmail.com

Published by the American Physical Society under the terms of the Creative Commons Attribution 4.0 International license. Further distribution of this work must maintain attribution to the author(s) and the published article's title, journal citation, and DOI. Funded by SCOAP ${ }^{3}$. scenarios, the extended scalar sector manifests from the Goldstone bosons, and unbroken symmetry does contain the SM electroweak group $S U(2) \times U(1)$. These sets of models, where the Higgs boson emerges as a "little" part of a bigger representation and where some global symmetry is broken by the interplay between two or more coupling constants, are termed little Higgs (LH) models [3], and many different variations of these models have been proposed in literature [5-8]. This collective symmetry breaking is the essential ingredient in the little Higgs theories in order to free the Higgs mass squared from quadratic divergences at one loop. On the other hand, some models, where the enlarged global symmetry is broken by some strong dynamics and the Higgs emerges as the pseudo-Nambu-Goldstone boson of that enlarged global symmetry, are termed composite Higgs models [9-12]. Here, one has to assume partial compositeness [13] of the $\mathrm{SM}$ fermions in order to generate their masses.

Due to the presence of the Nambu-Goldstone bosons (NGB) in the little Higgs theories [14,15] and the composite Higgs models [16-19], it is natural that they can usher in an extended scalar sector at the $\mathrm{TeV}$ scale. Some scenarios among these models represent an effective two-Higgs-doublet model (2HDM) [20] framework at the lower energy scale $(\sim \mathrm{TeV})$. We take particular interest in 
studying the case where the global symmetry $S U(6)$ is broken to $S p(6)$ to provide an extended LH group structure $S U(6) / S p(6)$, which is one variation of the little Higgs scenario developed by Low, Skiba, and Smith (LSS model) [21]. Similarly, there are composite Higgs models based on the same group structure $S U(6) / S p(6)[16,17]$, where, similarly to the little Higgs model, the low-energy effective theory exhibits a 2HDM structure, and in addition, beyondStandard-Model (BSM) particles exist. Even though the structures of these two models are alike, there are some differences between these two sets of models. The gauged subgroup in the $S U(6) / S p(6)$ little Higgs model is more extended than the electroweak (EW) subgroup, and the additional gauge generators are obtained via symmetry breaking at the condensation scale $(f)$. Also, the technical difference in the little Higgs and composite Higgs scenarios is the generation of the scalar potential terms with different quartic terms due to the nonidentical Yukawa structures of these two models. Hence, the dependencies of the emergent 2HDM scalar potential coefficients on different sets of model parameters are not similar in both cases. In this work, we choose to explore the LSS model with an emergent 2HDM structure at low energy in the view of recent flavor and LHC data.

In addition to the extended scalar sector, the BSM sector of the LSS model includes new fermions, such as one vectorlike quark doublet and two vectorlike quark singlets along with new gauge bosons. Natural realization of the LSS model in the low-energy effective theory can be executed through the alignment limit of the emergent 2HDM [21,22]. In the familiar 2HDM [20], the Yukawa sector is organized arbitrarily using the $Z_{2}$ symmetry according to the phenomenological requirements, whereas in the little Higgs models, a larger symmetry dictates it. On the other hand, in the LSS scenario, the Yukawa sector, which is initially arranged in terms of the fermionic multiplets aka sextets, directs the pattern of the Yukawa sector in emergent 2HDM after the symmetry breaking. Moreover, ultraviolet completion of the 2HDM is possible through the LSS model with an extended gauge sector $S U(6) / S p(6)$. Therefore, a study of this model can reveal effects of larger symmetry on the experimental observables that are unlikely to manifest in ad hoc generic 2HDM. A comprehensive phenomenological analysis of the LSS model is shown in Ref. [22], considering $8 \mathrm{TeV}$ LHC results. But the extended scalar sector of the LSS model demands a detailed study of collider phenomenology as well as the effects of BSM scalars in flavor physics observables. In this paper, we discuss the effective $2 \mathrm{HDM}$ emerging from the LSS model at low energy in the context of flavor and electroweak (EW) observables, along with constraints from $13 \mathrm{TeV}$ LHC.

In light of the significant tension between the SM and experimental measurements of lepton-flavor universality (LFU) observables [23], it is worthwhile to look for a
New Physics (NP) model, capable of explaining the discrepancies. In the LH models, the presence of new particles with possible nontrivial flavor structure can potentially address the challenges in the flavor sector. The flavor structure of different little Higgs models had been studied in the literature through multiple variants [2426], along with some phenomenological studies [27]. The major focus of these models had been to probe the effects of vectorlike fermions and the gauge bosons, significantly modifying the flavor observables. The SM extensions with two Higgs doublets, such as in Refs. [28,29] and various other scalar extensions of the SM [30,31], can also address the flavor anomalies with the presence of the extra scalars in the theory. This motivates us to particularly investigate the effect of the additional scalars of the LSS model in the flavor sector. It is worthwhile to study how the neutral and the charged BSM scalars can modify the flavor and other EW observables, and therefore impose constraints on the emergent 2HDM parameters derived from the LSS little Higgs model. At first, we assess the impact of different flavor observables and the $Z b \bar{b}$ vertex correction. Among all BSM scalars present, we take particular interest in studying the couplings of the charged Higgs to the thirdgeneration quarks to estimate the modifications in specific flavor observables. We observe that the $B$-meson mixing, $B$-meson decays, and correction to the $Z b \bar{b}$ vertex impose important constraints on the model parameter space.

In the LSS little Higgs model, the lightest $C P$-even neutral state is identified with $125 \mathrm{GeV}$ Higgs particles, observed at the LHC. Precise measurements of the top and bottom masses along with other measurements in the Higgs sector place nontrivial constraints on the model parameter space. Nonobservation of new states at the LHC $[32,33]$ and the EW precision tests (EWPT) [34] place strong limits on the BSM sector, and hence also constrain the LSS model. We determine the allowed regions of $\tan \beta$ and charged Higgs mass in the emergent 2HDM structure of the LSS model as compared to a generic 2HDM and study the crucial phenomenological differences. In contrast to the generic 2HDM with specific Yukawa structure (type-I, type-II, type-III $2 \mathrm{HDM}$, etc.), where the flavor constraints play a major role in ruling out a large parameter space, in the emergent 2HDM of the LSS model, constraints from EW searches at the LHC are found to be more severe than those coming from the flavor and $Z b \bar{b}$. This is due to the predictive nature of the Yukawa sector in the LSS model that shrinks the parameter space mainly to fix the Higgs mass, top quark mass, and top quark Yukawa coupling.

In Sec. II, we describe the Yukawa interaction in the LSS model to obtain the couplings of the scalar to the SM and BSM fermions. Then we show how an effective 2HDM framework can be worked out in the LSS model, connecting the strong sector parameters of the LSS model to the entities of the emergent 2HDM. We also show how different Yukawa structures can be arranged in some limits 
of the bottom-quark Yukawa couplings. In Sec. III, we focus on the different constraints from flavor and EW observables in the 2HDM framework of the LSS model. First, we consider all the relevant flavor and EW observables, discussing their possible origin and effects. Then, we scan over the entire model parameter space of the LSS model, curving out the region favored by the flavor and EW observables. Next, we bring in the LHC measurements and discuss their superconstraining effects on the parameter space, which is already constrained from flavor and EW data. Finally, in Sec. IV, we chart out the relative importance of our results in comparison to a generic $2 \mathrm{HDM}$.

\section{LOW-SKIBA-SMITH (LSS) LITTLE HIGGS MODEL}

The little Higgs model proposed by Low, Skiba, and Smith—i.e., the LSS model [21]—exhibits a larger symmetry $S U(6)$ in the unbroken form, which is broken to a residual symmetry $S p(6)$ by the field condensates. The number of massless Goldstone bosons that are expected to appear due to the symmetry breaking is equal to the difference in the number of generators-i.e., $35-21=14$. The NGBs $\left(\pi^{a}\right)$ are contained in $\Sigma=e^{\left[i \pi^{a} X^{a} / f\right]}\langle\Sigma\rangle$, where $\langle\Sigma\rangle$ is the antisymmetric condensate and $X^{a}$ 's are the broken generators, as given in Ref. [21]. These Goldstone bosons obtain relatively small masses from the radiative corrections and therefore can be termed as pseudo-Nambu-Goldstone bosons (pNGBs). Eight of these pNGBs form two scalar doublets $\left(\phi_{1}\right.$ and $\left.\phi_{2}\right)$, which, at the $\mathrm{TeV}$ scale, are similar to the scalar doublets of general 2HDM by construction. One major difference between a general 2HDM and the emergent 2HDM in the LSS model is the appearance of the vectorlike fermions and gauge bosons in the LSS model. These BSM particles, along with the 2HDM structure in the LSS model, can significantly alter the BSM scalar phenomenology, as shown in earlier works [22,35]. In the following, we discuss the Yukawa couplings of the scalars and their effective 2HDM analysis. For a detailed description of the model, we refer to Refs. [21,22].

\section{A. Yukawa sector: Diagonalization of mass matrices}

The diverse nature of the Yukawa sector in the LSS model requires special attention, as it has immense potential to churn out intricacies of flavor and other EW physics. Here, construction of the Yukawa sector involves an extension beyond the SM electroweak symmetry breaking. In the LSS model, the $S U(6)$ symmetry is broken explicitly by the gauge and Yukawa couplings in multiple steps, termed as collective symmetry breaking. In the fermion sector, this is ensured by a special structure of the Yukawa couplings [21]. In this model, we mainly focus on the couplings of the scalars with the third generation of fermions. Also, in this study we have adopted a different nomenclature for the fields compared to Ref. [22], and hence we show the following steps for clarity. The Yukawa Lagrangian is

$$
\begin{aligned}
\mathcal{L}_{Y u k}= & y_{1} f\left(\begin{array}{llll}
Q^{\prime} & t^{\prime \prime} & \left(i \sigma^{2} Q\right)^{T} & 0
\end{array}\right)(\Sigma)^{*}\left(\begin{array}{c}
0 \\
t^{c}
\end{array}\right)+y_{2} f\left(\begin{array}{llll}
0 & 0 & Q^{T} & 0
\end{array}\right)(\Sigma)\left(\begin{array}{c}
i \sigma^{2} Q^{\prime c} \\
t^{\prime \prime c} \\
0 \\
b^{\prime \prime c}
\end{array}\right) \\
& -i y_{1 b} f\left(\begin{array}{cccc}
0 & 0 & Q^{T} & 0
\end{array}\right)(\Sigma)\left(\begin{array}{c}
0 \\
0 \\
0 \\
b^{c}
\end{array}\right)+i y_{2 b} f\left(\begin{array}{llll}
0 & 0 & \left(i \sigma_{2} Q\right)^{T} & 0
\end{array}\right)(\Sigma)^{*}\left(\begin{array}{c}
0 \\
b^{c} \\
0 \\
0
\end{array}\right)+\text { H.c. }
\end{aligned}
$$

The new fermions are one vectorlike quark doublet Weyl-fermion pair $Q^{\prime}=\left(t^{\prime}, b^{\prime}\right)^{T}, Q^{\prime c}=\left(-b^{\prime c}, t^{\prime c}\right)^{T}$ with $Y=1 / 6$ and EM charge $2 / 3$, one vectorlike up-type quark singlet $t^{\prime \prime}, t^{\prime \prime c}$ with EM charge $\pm 2 / 3$, and one vectorlike down-type quark singlet $b^{\prime \prime}, b^{\prime \prime c}$ with EM charge $\mp 1 / 3 . Q=(t, b)^{T}$ is the SM SU(2) doublet. $y_{i}$ and $y_{i b}$ (for $i=1,2$ ) are the dimensionless couplings in the top and bottom sectors, respectively. Expanding the SU(2) structure of the Yukawa couplings and including the vectorlike fermion masses, we get

$$
\begin{aligned}
\mathcal{L}_{\text {mass }+ \text { Yuk }} \supset & -y_{1}\left(f t^{\prime \prime} t^{c}-i Q^{\prime T} \phi_{2}^{*} t^{c}-i Q^{T} \cdot \phi_{1} t^{c}\right)+y_{2}\left(f Q^{T} \cdot Q^{\prime c}+i Q^{T} \boldsymbol{\phi}_{1}^{*} b^{\prime \prime c}+i Q^{T} \boldsymbol{\phi}_{2}^{*} t^{\prime \prime c}\right) \\
& +y_{3} f\left(Q^{\prime T} \cdot Q^{\prime c}\right)+y_{4} f\left(t^{\prime \prime c} t^{\prime \prime}\right)+y_{5} f\left(b^{\prime \prime c} b^{\prime \prime}\right)+y_{1 b}\left(Q^{T} \boldsymbol{\phi}_{1}^{*} b^{c}\right)-y_{2 b}\left(Q^{T} \cdot \phi_{2} b^{c}\right)+\text { H.c. }
\end{aligned}
$$

Here $y_{3}, y_{4}$, and $y_{5}$ are also dimensionless constants. From Eq. (2), we can infer the fermion mass matrices after electroweak symmetry breaking (EWSB). The fermion mass matrices with EM charges $+2 / 3$ and $-1 / 3$ appear in the Lagrangian as 


$$
\mathcal{L} \supset\left(\begin{array}{lll}
t & t^{\prime} & t^{\prime \prime}
\end{array}\right)\left(\begin{array}{ccc}
i y_{1} \frac{v_{1}}{\sqrt{2}} & y_{2} f & i y_{2} \frac{v_{2}}{\sqrt{2}} \\
i y_{1} \frac{v_{2}}{\sqrt{2}} & y_{3} f & 0 \\
-y_{1} f & 0 & y_{4} f
\end{array}\right)\left(\begin{array}{c}
t^{c} \\
t^{\prime c} \\
t^{\prime \prime c}
\end{array}\right)+\left(\begin{array}{lll}
b & b^{\prime} & b^{\prime \prime}
\end{array}\right)\left(\begin{array}{ccc}
y_{i b} \frac{v_{i}}{\sqrt{2}} & y_{2} f & i y_{2} \frac{v_{1}}{\sqrt{2}} \\
0 & y_{3} f & 0 \\
0 & 0 & y_{5} f
\end{array}\right)\left(\begin{array}{c}
b^{c} \\
b^{\prime c} \\
b^{\prime \prime c}
\end{array}\right)+\text { H.c., }
$$

where $v_{i}=\left\{v_{1}, v_{2}\right\}$ are the vacuum expectation values (VEVs) of $\phi_{1}$ and $\phi_{2}$, respectively. After diagonalizing Eq. (3), we get couplings of the scalars to the fermions for the top sector, in their mass basis. We implement a two-step diagonalization, where first the $f$-dependent terms are diagonalized analytically, and then the $v_{1,2}$-dependent terms (which appear after EWSB) are diagonalized. We define the rotations that diagonalize the $f$-dependent terms as

$$
\left(\begin{array}{c}
t \\
t^{\prime} \\
t^{\prime \prime}
\end{array}\right)=\left(\begin{array}{ccc}
c_{23} & -s_{23} & 0 \\
s_{23} & c_{23} & 0 \\
0 & 0 & 1
\end{array}\right)\left(\begin{array}{l}
t_{0} \\
t_{1} \\
t_{2}
\end{array}\right) ;\left(\begin{array}{c}
t^{c} \\
t^{\prime c} \\
t^{\prime \prime c}
\end{array}\right)=\left(\begin{array}{ccc}
c_{14} & 0 & -s_{14} \\
0 & 1 & 0 \\
s_{14} & 0 & c_{14}
\end{array}\right)\left(\begin{array}{l}
t_{0}^{c} \\
t_{1}^{c} \\
t_{2}^{c}
\end{array}\right) ;\left(\begin{array}{c}
b \\
b^{\prime} \\
b^{\prime \prime}
\end{array}\right)=\left(\begin{array}{ccc}
c_{23} & -s_{23} & 0 \\
s_{23} & c_{23} & 0 \\
0 & 0 & 1
\end{array}\right)\left(\begin{array}{l}
b_{0} \\
b_{1} \\
b_{2}
\end{array}\right),
$$

with $s_{23} \equiv \sin \theta_{23}=y_{2} /\left(\sqrt{y_{2}^{2}+y_{3}^{2}}\right), c_{23} \equiv \cos \theta_{23}=-y_{3} /\left(\sqrt{y_{2}^{2}+y_{3}^{2}}\right)$, and $s_{14} \equiv \sin \theta_{14}=y_{1} /\left(\sqrt{y_{1}^{2}+y_{4}^{2}}\right)$. After these rotations, some field redefinitions are performed to make the mass matrix entries real and positive. ${ }^{1}$ For brevity of notation, tildes on the fields are dropped, and we denote the field $\tilde{\chi}_{i}$ simply as $\chi_{i}$ in the following. Hence, the mass matrices in the Lagrangian take the form

$$
\mathcal{L}^{\text {mass }} \supset\left(\begin{array}{lll}
t_{0} & t_{1} & t_{2}
\end{array}\right)\left(\begin{array}{ccc}
\mathcal{M}_{11}^{t} & 0 & \mathcal{M}_{13}^{t} \\
\mathcal{M}_{21}^{t} & \mathcal{M}_{22}^{t} & \mathcal{M}_{23}^{t} \\
0 & 0 & -\mathcal{M}_{33}^{t}
\end{array}\right)\left(\begin{array}{c}
t_{0}^{c} \\
t_{1}^{c} \\
t_{2}^{c}
\end{array}\right)+\left(\begin{array}{lll}
b_{0} & b_{1} & b_{2}
\end{array}\right)\left(\begin{array}{ccc}
\mathcal{M}_{11}^{b} & 0 & \mathcal{M}_{13}^{b} \\
\mathcal{M}_{21}^{b} & \mathcal{M}_{22}^{b} & \mathcal{M}_{23}^{b} \\
0 & 0 & \mathcal{M}_{33}^{b}
\end{array}\right)\left(\begin{array}{l}
b_{0}^{c} \\
b_{1}^{c} \\
b_{2}^{c}
\end{array}\right)+\text { H.c. }
$$

After the field redefinitions, the new mass matrix entries $\mathcal{M}_{i j}^{t, b}$ are

$$
\begin{aligned}
& \mathcal{M}_{11}^{t}=\frac{y_{1}\left(y_{3} y_{4} v_{1}+y_{2} y_{3} v_{2}-y_{2} y_{4} v_{2}\right)}{\sqrt[y]{14}_{14} \sqrt{y}_{23} \sqrt{2}}, \quad \mathcal{M}_{13}^{t}=\frac{\left(y_{1}^{2} y_{3} v_{1}-y_{2} y_{3} y_{4} v_{2}-y_{1}^{2} y_{2} v_{2}\right)}{\sqrt{y}_{14} \sqrt{y}_{23} \sqrt{2}}, \\
& \mathcal{M}_{21}^{t}=\frac{y_{1}\left(y_{2} y_{4} v_{1}+y_{2}^{2} v_{2}+y_{3} y_{4} v_{2}\right)}{\sqrt{y}_{14} \sqrt{y}_{23} \sqrt{2}}, \quad \mathcal{M}_{23}^{t}=\frac{\left(y_{1}^{2} y_{2} v_{1}-y_{2}^{2} y_{4} v_{2}+y_{1}^{2} y_{3} v_{2}\right)}{\sqrt{y}_{14} \sqrt{y}_{23} \sqrt{2}} \\
& \mathcal{M}_{33}^{t}=-f \sqrt{y}_{14}, \quad \mathcal{M}_{22}^{t}=f \sqrt{y}_{23}, \\
& \mathcal{M}_{11}^{b}=c_{23} y_{i b} \frac{v_{i}}{\sqrt{2}}, \quad \mathcal{M}_{13}^{b}=y_{2} \frac{v_{1}}{\sqrt{2}}, \quad \mathcal{M}_{33}^{b}=y_{5} f, \\
& \mathcal{M}_{21}^{b}=y_{i b} \frac{v_{i}}{\sqrt{2}} s_{23}, \quad \mathcal{M}_{23}^{b}=-y_{2} \frac{v_{2}}{\sqrt{2}} s_{23}, \quad \mathcal{M}_{22}^{b}=\sqrt{y}_{23} f,
\end{aligned}
$$

where $\sqrt{y}_{14} \equiv \sqrt{y_{1}^{2}+y_{4}^{2}}, \sqrt{y}_{23} \equiv \sqrt{y_{2}^{2}+y_{3}^{2}}$.

In the next step, we diagonalize the $v_{1,2}$-dependent terms of the matrix $\mathcal{M}_{i j}^{t}$, where the entries of $\mathcal{M}_{i j}^{t}$ are functions of intrinsic parameters of the $S U(6) / S p(6)$ LSS model. This is achieved through carrying out a biorthogonal transformation, constructing left and right orthogonal matrices $R_{L}$ and $R_{R}$, such that

$$
R_{L} \mathcal{M}_{i j}^{t} R_{R}^{T} \equiv\left(\mathcal{M}_{i j}^{t}\right)_{D}
$$

where $\left(\mathcal{M}_{i j}^{t}\right)_{D}$ is the diagonal matrix in the mass basis. Instead of showing explicit dependences of $R_{L}, R_{R}$, we perform the diagonalization along with the parameter space scan, where for each set of input parameters the diagonalization process is being performed. This process is iterated multiple times, keeping the parameter dependence of these matrices. The transformations of the fields are defined as

$$
\left(\begin{array}{lll}
t_{0} & t_{1} & t_{2}
\end{array}\right)^{T}=R_{L}^{T}\left(\begin{array}{lll}
T_{2} & T_{1} & t
\end{array}\right)^{T}, \quad\left(\begin{array}{ccc}
t_{0}^{c} & t_{1}^{c} & t_{2}^{c}
\end{array}\right)^{T}=R_{R}^{T}\left(\begin{array}{ccc}
T_{2}^{c} & T_{1}^{c} & t^{c}
\end{array}\right)^{T}
$$

${ }^{1}$ The field redefinitions are used, following the notation in Ref. [22]: $t_{0}^{c}=i \tilde{t}_{0}^{c}, t_{1}^{c}=-i \tilde{t}_{1}^{c}, t^{\prime c}=-\tilde{t}^{\prime c}, \psi_{1}=i \tilde{\psi}_{1}$, with $b_{1}=-\tilde{b}_{1}$, $\psi_{2}^{c}=-i \tilde{\psi}_{2}^{c}$, and $\psi_{2}=i \tilde{\psi}_{2}$. 
where $T_{i}, t$ and $T_{i}^{c}, t^{c}$ are the mass eigenstates in the top sector. We identify $\left(t, t^{c}\right)$ as the observed top-quark $(t)$ mass eigenstates, and the others correspond to the mass eigenstates of the vectorlike top partners $\left(T_{1}, T_{2}\right)$. In the bottom sector, the off-diagonal terms proportional to $v_{i}$ 's are numerically insignificant due to the smallness of $y_{i b}$, and hence we do not perform the mass diagonalization. We identify $\left(b_{0}, b_{0}^{c}\right)$ as the observed bottom quark $(b)$, and the others correspond to vectorlike bottom partners $\left(B_{1}, B_{2}\right)$. We also identify the mass eigenvalues as $m_{t}, M_{T_{1}}, M_{T_{2}}$ in the top sector, and $m_{b}, M_{B_{1}}, M_{B_{2}}$ in the bottom sector. In order to identify the eigenvalues corresponding to $m_{t}, M_{T_{1}}$, $M_{T_{2}}$ and $m_{b}, M_{B_{1}}, M_{B_{2}}$, it is ensured that $m_{t}<M_{T_{1}}<M_{T_{2}}$ and $m_{b}<M_{B_{1}}<M_{B_{2}}$.

\section{B. Yukawa couplings of the scalars}

In this section, we are mainly interested in couplings of the SM-like Higgs $h$ and the charged Higgs $H^{ \pm}$to the fermions, because those couplings affect the flavor and EW observables the most, as far as the model parameter space of the LSS model is concerned. We will discuss this further at the end of this section. In the following, we present the structure of the relevant Yukawa couplings-i.e., the couplings of $h$ and $H^{ \pm}$-in detail.

Before the mass diagonalization, the top-quark Yukawa coupling with a Higgs-like scalar $(h)$ in the flavor basis can be written as

$\mathcal{L}_{h t t}=\frac{i h}{\sqrt{2}}\left(\begin{array}{lll}t & \psi_{1} & t^{\prime}\end{array}\right)\left(\begin{array}{ccc}y_{1} c_{\alpha} & -y_{2} s_{\alpha} & 0 \\ 0 & 0 & 0 \\ -y_{1} s_{\alpha} & 0 & 0\end{array}\right)\left(\begin{array}{c}t^{c} \\ \psi_{1}^{c} \\ t^{\prime c}\end{array}\right)+$ H.c.

Then we rewrite the Lagrangian in the basis where the $f$ terms are diagonal. ${ }^{2}$ Also, we implement the field redefinitions as stated previously to render the real fermion mass matrix. Hence, the Yukawa coupling of $h$ on an intermediate basis now takes the form

$\mathcal{L}_{h}^{\text {Yuk }} \supset \frac{h}{\sqrt{2}}\left[y_{00} t_{0} t_{0}^{c}+y_{01} t_{0} t_{1}^{c}+y_{10} t_{1} t_{0}^{c}+y_{11} t_{1} t_{1}^{c}\right]+$ H.c.,

with $\quad y_{00} \equiv\left(-y_{1} c_{\alpha} c_{14} c_{23}+y_{1} s_{\alpha} c_{14} s_{23}+y_{2} s_{\alpha} s_{14} c_{23}\right)$, $y_{01} \equiv\left(-y_{1} c_{\alpha} s_{14} c_{23}+y_{1} s_{\alpha} s_{14} s_{23}-y_{2} s_{\alpha} c_{14} c_{23}\right), \quad y_{10} \equiv$ $\left(y_{1} c_{\alpha} c_{14} s_{23}+y_{1} s_{\alpha} c_{14} c_{23}-y_{2} s_{\alpha} s_{14} s_{23}\right), \quad$ and $\quad y_{11} \equiv$ $\left(y_{1} c_{\alpha} s_{14} s_{23}+y_{1} s_{\alpha} s_{14} c_{23}+y_{2} s_{\alpha} c_{14} s_{23}\right)$. Yukawa couplings

\footnotetext{
${ }^{2}$ In our convention, we define the top-quark Yukawa coupling $y_{h t t}$ as $\mathcal{L}_{h t t}=(h / \sqrt{2}) y_{h t t} \hat{t}_{0} \hat{t}_{0}^{c}+$ H.c. We define this with a positive sign here, since our field redefinitions make the fermion mass terms positive.
}

after the biorthogonal rotations to the mass basis, as defined in Eq. (8), take the form

$$
\begin{aligned}
y_{h t t}= & {\left[y_{00}\left(R_{L}\right)_{31}\left(R_{R}\right)_{31}+y_{01}\left(R_{L}\right)_{31}\left(R_{R}\right)_{32}\right.} \\
& \left.+y_{10}\left(R_{L}\right)_{32}\left(R_{R}\right)_{31}+y_{11}\left(R_{L}\right)_{32}\left(R_{R}\right)_{32}\right],
\end{aligned}
$$

where $\left(R_{L}\right)_{i j}$ and $\left(R_{R}\right)_{i j}$, with $i, j=\{1,2,3\}$, are the $(i, j)$ th entries of the rotation matrices $R_{L}$ and $R_{R}$, respectively. We define the deviation of $h t t$ from its SM value as

$$
k_{t t h} \equiv y_{h t t} / y_{h t t}^{\mathrm{SM}}
$$

where $y_{h t t}^{\mathrm{SM}}=m_{t} / v$. Similarly, Yukawa couplings of the $C P$-even neutral scalar $(H)$ can be obtained from Eq. (10) by making the change $c_{\alpha} \rightarrow s_{\alpha}$ and $s_{\alpha} \rightarrow-c_{\alpha}$. For the couplings of the pseudoscalar $(A)$, we refer to Ref. [22].

The Yukawa coupling of the $b$ quark with $h$ and the mass of the $b$ quark can be derived from Eq. (2) as

$\mathcal{L}_{h b b} \supset \frac{c_{23}}{\sqrt{2}}\left[v\left(y_{1 b} s_{\beta}+y_{2 b} c_{\beta}\right)+h\left(y_{1 b} c_{\alpha}-y_{2 b} s_{\alpha}\right)\right] b_{0} b^{c}+$ H.c.

The coupling and the mass are thus identified to be

$$
m_{b} \equiv v\left(y_{1 b} s_{\beta}+y_{2 b} c_{\beta}\right) / \sqrt{2}, \quad y_{h b b} \sim m_{b} / v .
$$

Analogous mass and coupling expressions hold for the third-generation lepton $(\tau)$ with the replacement $y_{b} \rightarrow y_{\tau}$.

Similarly, couplings of the charged Higgs with the thirdgeneration quarks can be obtained as

$$
\begin{aligned}
\mathcal{L}_{H^{ \pm}}^{Y u k} \supset & H^{+}\left(y_{00}^{+} b_{0} t_{0}^{c}+y_{01}^{+} b_{0} t_{1}^{c}+y_{10}^{+} b_{1} t_{0}^{c}+y_{11}^{+} b_{1} t_{1}^{c}\right) \\
& +H^{-}\left(y_{00}^{-} t_{0} b^{c}+y_{10}^{-} t_{1} b^{c}+y_{02}^{-} t_{0} \psi_{2}^{c}+y_{12}^{-} t_{1} \psi_{2}^{c}\right) \\
& + \text { H.c. }
\end{aligned}
$$

where

$$
\begin{aligned}
& y_{00}^{+}=\left(y_{1} s_{\beta} s_{23} c_{14}-y_{1} c_{\beta} c_{23} c_{14}+y_{2} s_{\beta} c_{23} s_{14}\right), \\
& y_{01}^{+}=\left(y_{1} s_{\beta} s_{23} s_{14}-y_{1} c_{\beta} c_{23} s_{14}-y_{2} s_{\beta} c_{23} c_{14}\right), \\
& y_{10}^{+}=\left(-y_{1} s_{\beta} c_{23} c_{14}-y_{1} c_{\beta} s_{23} c_{14}+y_{2} s_{\beta} s_{23} s_{14}\right), \\
& y_{11}^{+}=\left(-y_{1} s_{\beta} c_{23} s_{14}-y_{1} c_{\beta} s_{23} s_{14}-y_{2} s_{\beta} s_{23} c_{14}\right), \\
& y_{00}^{-}=\left[\begin{array}{ll}
\left.\left(-y_{1 b} c_{\beta}+y_{2 b} s_{\beta}\right) c_{23}\right], \quad y_{10}^{-}=\left[\left(y_{1 b} c_{\beta}-y_{2 b} s_{\beta}\right) s_{23}\right.
\end{array}\right], \\
& y_{02}^{-}=\left(-y_{2} c_{\beta} c_{23}\right), \quad y_{12}^{-}=\left(y_{2} c_{\beta} s_{23}\right) .
\end{aligned}
$$

After the numerical computation of the rotation matrices $\left(R_{L}, R_{R}\right)$ that diagonalize the off-diagonal terms proportional to $v_{1,2}$, we obtain the Lagrangian involving the charged Higgs couplings with top and bottom quarks as 
$\mathcal{L}_{H^{ \pm} t b} \supset V_{t b} \frac{1}{v} H^{+} \bar{t}\left(y_{H^{ \pm} t_{L} b_{R}} m_{t} P_{R}+y_{H^{ \pm} t_{R} b_{L}} m_{b} P_{L}\right) b+$ H.c.,

where the charged Higgs Yukawa couplings are given by

$$
\begin{aligned}
& y_{H^{ \pm} t_{L} b_{R}}=\left[y_{00}^{-}\left(R_{L}\right)_{31}+y_{10}^{-}\left(R_{L}\right)_{32}\right], \\
& y_{H^{ \pm} t_{R} b_{L}}=\left[y_{00}^{+}\left(R_{R}\right)_{31}+y_{01}^{+}\left(R_{R}\right)_{32}\right] .
\end{aligned}
$$

The relevant Lagrangian involving the coupling of top and strange quarks with the charged Higgs can be obtained in a similar way:

$\mathcal{L}_{H^{ \pm} t s} \supset V_{t s} \frac{1}{v} H^{+} \bar{t}\left(y_{H^{ \pm} t_{L} s_{R}} m_{t} P_{R}+y_{H^{ \pm} t_{R} s_{L}} m_{s} P_{L}\right) s+$ H.c.,

where $y_{H^{ \pm} t_{L} s_{R}}, y_{H^{ \pm} t_{R} s_{L}}$ can be derived from Eq. (16) with the replacement of $y_{1 b} \rightarrow y_{1 s}, y_{2 b} \rightarrow y_{2 s}$ and then used analogously to the $y_{00}^{-}, y_{10}^{-}$terms in Eq. (17). Note that the first parts of both the couplings are similar, and being proportional to $m_{t}$, they are the dominant terms. The second parts of the Lagrangian, being proportional to $m_{b}$ or $m_{s}$, are not significant due to the smallness of the masses. Moreover, the couplings are of the same order-i.e., $y_{1 b} \sim y_{1 s}, y_{2 b} \sim y_{2 s}$. The coupling involving the down quark, $H^{ \pm} t d$, will also hold to a similar expression, but the coupling is relatively small due to the smallness of $V_{t d}$. Overall, ${ }^{3}$ the couplings $H^{ \pm} t d$ and $H^{ \pm} t s$ will be smaller compared to $H^{ \pm} t b$.

Among the other couplings of the charged Higgs, the $H^{ \pm} c s$ and $H^{ \pm} \tau \nu$ couplings can be obtained as

$$
\begin{aligned}
\mathcal{L}^{\text {Yuk }} \supset & \left(y_{1 c} c_{\beta}-y_{2 c} s_{\beta}\right) H^{+} s c^{c}+\left(-y_{1 s} c_{\beta}+y_{2 s} s_{\beta}\right) H^{-} c s^{c} \\
& +\left(-y_{1 \tau} c_{\beta}+y_{2 \tau} s_{\beta}\right) H^{-} \nu \tau^{c}+\text { H.c. }
\end{aligned}
$$

The charged Higgs decays dominantly through the $H^{ \pm} \rightarrow t b$ mode for most of the parameter space, unless there is an abrupt cancellation between different contributions of Eq. (16) [22]. Otherwise, the second most prominent decay mode, $H^{ \pm} \rightarrow c s$, takes over. The leptonic decay mode $H^{ \pm} \rightarrow \tau^{ \pm} \nu$ is not found to be very significant. Hence, the tree-level charged-current contributions from the diagrams containing $H^{ \pm}$are negligible in this model.

The couplings of the neutral pseudoscalar $(A)$ and the heavy scalar $(H)$ are studied in detail in Ref. [22], which shows that $H$ and $A$ couple to third-generation quarks strongly. Their couplings to the leptons, as well as to the first and second generations of quarks, are negligible. Hence, tree-level FCNC processes in meson decays, neutral

\footnotetext{
${ }^{3}$ The couplings with lighter fermions that we neglect here appear in squares in the flavor observables, and their effect is much smaller compared to the anomalies present in the flavor observables.
}

meson mixing, and other lepton-flavor-violating decays are suppressed in this model. We discuss different structures of the Yukawa couplings in an effective 2HDM framework and their effects on FCNCs in the next section.

\section{Effective 2HDM framework of LSS model}

If the radiative corrections due to the presence of extra heavier gauge bosons, vectorlike quarks, and the singlet scalars are included in the LSS model, an effective scalar potential at the $\mathrm{TeV}$ scale mimics that of the $2 \mathrm{HDM}$, albeit with the absence of a number of scalar field combinations. The scalar potential generated at one loop in the LSS model [21] is

$$
\begin{aligned}
\mathcal{V}_{\mathrm{LSS}}= & m_{1}^{2}\left|\phi_{1}\right|^{2}+m_{2}^{2}\left|\phi_{2}\right|^{2}+\left(b^{2} \phi_{1}^{T} \cdot \phi_{2}+\text { H.c. }\right) \\
& +\lambda_{5}^{\prime}\left|\phi_{1}^{T} \cdot \phi_{2}\right|^{2},
\end{aligned}
$$

where $\phi_{1}$ and $\phi_{2}$ are the $S U(2)$ scalar doublets with hypercharges $+1 / 2$ and $-1 / 2$, respectively, and $\phi_{1}^{T}$. $\phi_{2}=$ $\phi_{1}^{T} i \sigma^{2} \phi_{2}$ is the antisymmetric product of the fields. Similar to the 2HDM case, the ratio of the VEVs of the scalar doublets $\phi_{1,2}$ is presented in terms of

$$
\tan \beta \equiv v_{1} / v_{2}=\sqrt{m_{2}^{2} / m_{1}^{2}},
$$

which is a function of the LSS scalar potential parameters $m_{i}^{2}$. The physical masses of the scalars that constitute the 2HDM structure can be expressed as

$$
\begin{aligned}
m_{A}^{2} & =2 b^{2} / \sin (2 \beta), \quad m_{H^{ \pm}}^{2}=m_{A}^{2}-\lambda_{5}^{\prime} v^{2} / 2, \\
m_{H, h}^{2} & =\frac{1}{2}\left[m_{A}^{2} \pm \sqrt{m_{A}^{4}-4\left(m_{A}^{2}-m_{H^{ \pm}}^{2}\right) m_{H^{ \pm}}^{2} \sin ^{2}(2 \beta)}\right] .
\end{aligned}
$$

The parameters $m_{1}^{2}, m_{2}^{2}, b^{2}$, and $\lambda_{5}^{\prime}$ are functions of the $S U(6) / S p(6)$-model Lagrangian parameters and can be expressed as

$$
\begin{aligned}
\lambda_{5}^{\prime} & =\frac{\left.c g_{1}^{2}\left[g_{2}^{2}+\left(c^{\prime} / c\right) y_{2}^{2}\right]\right)}{g_{1}^{2}+g_{2}^{2}+\left(c^{\prime} / c\right) y_{2}^{2}} \\
b^{2} & =\frac{3 f^{2}}{8 \pi^{2}} y_{1}^{2} y_{2}\left(y_{3}-y_{4}\right) \log \frac{\Lambda^{2}}{M_{f}^{2}}, \\
m_{1 f}^{2} & =\frac{3 f^{2}}{8 \pi^{2}}\left(y_{1}^{2}-y_{2}^{2}\right)\left(y_{3}^{2}-y_{4}^{2}\right) \log \frac{\Lambda^{2}}{M_{f}^{2}}, \\
m_{2 f}^{2} & =\frac{3 f^{2}}{8 \pi^{2}}\left(y_{1}^{2} y_{2}^{2}+y_{2}^{2} y_{5}^{2}-y_{2}^{2} y_{3}^{2}-y_{1}^{2} y_{4}^{2}\right) \log \frac{\Lambda^{2}}{M_{f}^{2}}, \\
m_{1 g}^{2} & =m_{2 g}^{2}=\frac{3}{64 \pi^{2}}\left[3 g^{2} M_{g}^{2} \log \frac{\Lambda^{2}}{M_{g}^{2}}+g^{\prime 2} M_{g^{\prime}}^{2} \log \frac{\Lambda^{2}}{M_{g^{\prime}}^{2}}\right] \\
m_{1 s}^{2} & =m_{2 s}^{2}=\frac{\lambda_{5}^{\prime}}{16 \pi^{2}} M_{s}^{2} \log \frac{\Lambda^{2}}{M_{S}^{2}} .
\end{aligned}
$$


$\lambda_{5}^{\prime}$ gets gauge (proportional to $g_{1}$ and $g_{2}$ ) as well as fermionic (proportional to $y_{2}$ ) contributions, whereas $b^{2}$ receives only the fermionic contribution (proportional to $y_{i}$ ). In Eq. (23), $m_{1}^{2}$ and $m_{2}^{2}$ get contributions from the scalars $(s)$, gauge bosons $(g)$, and fermions $(f)$ in the loop. The degrees of constructive or destructive interference depend on the signs of the coefficients. $\Lambda$ is the cutoff, which is taken to be $4 \pi f$, where $f$ is the energy scale associated with $S U(6) / S p(6)$ breaking. $M_{f}$ is the heavy vectorlike fermion mass scale. The heavy gauge boson masses are $M_{g}=f \sqrt{\left(g_{1}^{2}+g_{2}^{2}\right) / 2}$ and $M_{g^{\prime}}=f \sqrt{\left(g_{1}^{\prime 2}+g_{2}^{\prime 2}\right) / 2}$. The singlet scalar mass is $M_{s}=f \sqrt{c\left(g_{1}^{2}+g_{2}^{2}\right)+c^{\prime} y_{2}^{2}}$, where $c$ and $c^{\prime}$ are $O(1)$ parameters that depend on the details of UV completion, as explained in Ref. [22].

How the two scalar doublets $\phi_{1}$ and $\phi_{2}$ couple to other lighter fermions (lighter than the top) in the 2HDM determines the presence or absence of FCNCs in the theory. As tree-level FCNCs are tightly bound by experimental absence, they can place stringent constraints on the model. The tree-level FCNCs are absent in this emergent 2HDM from the LSS model, if the $Z_{2}$ symmetry is not broken. Symmetries in the LSS model compel the top quark to couple to both $\phi_{1}$ and $\phi_{2}$ [see Eq. (1)], which breaks the $Z_{2}$ symmetry of the $2 \mathrm{HDM}$ in the top sector. This type of model, with Type III 2HDM flavor structure, results in nontrivial FCNCs in the third generation.

In the light fermionic Yukawa sector, $Z_{2}$ symmetry can be enforced-i.e., either $\phi_{1}$ or $\phi_{2}$ couples to the fermions. With the Yukawa interactions $y_{1(b, \tau, c)} \neq 0$ and $y_{2(b, \tau, c)}=0$-i.e., light down-type fermions being coupled only to $\phi_{1}$-stringent constraints from the LHC such as $h \rightarrow b \bar{b}, \tau \bar{\tau}$ measurements become important. The alternate possibility is $y_{1(b, \tau, c)}=0$ and $y_{2(b, \tau, c)} \neq 0$, which relaxes the earlier constraints. If this Yukawa structure is adopted for the up-type light fermions as well, this will resemble a Type I 2HDM framework in the light fermion sector, while the top sector will break it. The earlier framework, $y_{1(b, \tau, c)} \neq 0$ and $y_{2(b, \tau, c)}=0$, along with the up-type coupling with $\phi_{1}$, is also a Type I framework for the light fermions, but that is not so tenable from the LHC constraints.

On the other hand, an alternative Yukawa pattern can be explored where $y_{1} \neq 0$ for the up-type light fermions, with them being coupled only to $\phi_{1}$ and the down-type fermions only coupled to $\phi_{2}$-i.e., $y_{2} \neq 0$ for the down type. The light fermion sector in this scenario will look like a Type II $2 \mathrm{HDM}$ setup. If the top couples to both $\phi_{1}$ and $\phi_{2}$, it breaks the Type II structure, as seen earlier. The constraints from the $h \rightarrow b \bar{b}, \tau \bar{\tau}$ at the LHC are relaxed in this scenario. Hence, these types of peculiarity in the flavor structure are potentially ripe for nontrivial implications in the flavor physics sector. A detailed analysis of the impact of a few hitherto important flavor observables in the $2 \mathrm{HDM}$ sector is the core of this work.

\section{CONSTRAINTS FROM FLAVOR AND ELECTROWEAK PHYSICS}

The LSS model can exhibit different Yukawa patterns, leading to different 2HDM-like scenarios. Depending on the flavor structure of the Yukawa couplings, flavorchanging neutral currents can place important constraints on the model. We study the FCNCs involving different quarks, among which the third-generation ones are in particular nontrivial. In this model, the top quark couples to both $\phi_{1}$ and $\phi_{2}$. The Yukawa coupling involving the bottom quark depends on the model parameters $y_{1 b}$ and $y_{2 b}$, which play a major role in the calculation of the flavor observables. The choice of $y_{1 b} \neq 0, y_{2 b} \neq 0$ reflects the Type III 2HDM-like scenario. We have also considered alternative scenarios where $y_{1 b}=0, y_{2 b} \neq 0$ or $y_{1 b}=0$, $y_{2 b} \neq 0$, which can lead to different 2HDM scenarios, as mentioned in Sec. II C. ${ }^{4}$ Hence, in the LSS model we study three cases: Case I:

$$
\begin{array}{r}
\text { Case I: } y_{1 b} \neq 0, \quad y_{2 b} \neq 0 . \\
\text { Case II: } y_{1 b}=0, \quad y_{2 b} \neq 0 . \\
\text { Case III: } y_{1 b} \neq 0, \quad y_{2 b}=0 .
\end{array}
$$

\section{A. Flavor observables and $Z \boldsymbol{Z} \bar{b}$}

As reflected in the previous section, the effect of the Yukawa couplings involving the third-generation quarks has its dominant contribution in flavor and EW observables. Also, as mentioned earlier, the contribution of the tree-level FCNC can be avoided in a large model parameter space of the LSS model. Hence, while discussing the FCNCs, we will be focusing only on the loop-level FCNCs in the following text. Note that the vectorlike fermions also have significant impact on the flavor and EW observables [37], but in this article we focus on the scalar sector of the effective 2HDM in the LSS model. We also include constraints from $Z b \bar{b}$ measurement, while the $S$ and $T$ parameters are kept well within the limit by keeping the new gauge degrees of freedom on the heavier side [22]. We discuss different flavor observables, dividing them into a few broad categories.

\section{Radiative B-meson decays}

In the context of the 2HDM, the most stringent constraint comes from the radiative $B$-meson decays, $B \rightarrow X_{s} \gamma$ $\left(B \rightarrow X_{d} \gamma\right)$. The latest experimental value and the theoretical value of $\operatorname{BR}\left(B \rightarrow X_{s} \gamma\right)$ show discrepancy, as indicated in Table I. In the SM, the quark-level transition is mediated by $W$-boson and $t$-quark exchange via an electromagnetic penguin diagram. The matrix element for this process at the

\footnotetext{
${ }^{4}$ For a detailed study of the flavor structure of general 2HDM models, one might look at Ref. [36].
} 
TABLE I. Standard model vs experimental values of the flavor observables.

\begin{tabular}{lcc}
\hline \hline Observables & SM value & Experimental value \\
\hline $\operatorname{Br}\left(B \rightarrow X_{s} \gamma\right)$ & $(3.36 \pm 0.23) \times 10^{-4}[23]$ & $(3.32 \pm 0.16) \times 10^{-4}[38]$ \\
$\Delta m_{B_{s}}$ & $(17.757 \pm 0.021) \mathrm{ps}^{-1}[23]$ & $(18.3 \pm 2.7) \mathrm{ps}^{-1}[23]$ \\
$R_{b}$ & $0.21581 \pm 0.00011[39]$ & $0.21629 \pm 0.00066[34]$ \\
$B_{s} \rightarrow \mu \mu$ & $(3.65 \pm 0.23) \times 10^{9}[40]$ & $\left(3.0 \pm 0.6_{-0.2}^{+0.3}\right) \times 10^{9}[41]$ \\
\hline \hline
\end{tabular}

electroweak scale is governed by the dipole operator. The effective Hamiltonian for this process is given by

$$
\mathcal{H}_{\mathrm{eff}}=-\frac{4 G_{F}}{\sqrt{2}} V_{t s}^{*} V_{t b} \sum_{i=1}^{8} C_{i}(\mu) O_{i}(\mu) .
$$

Here, $V_{i j}$ represents the relevant Cabibbo-KobayashiMaskawa (CKM) factors. The $O_{i}$ 's are a complete set of renormalized dimension-six operators. They consist of six four-quark operators, $O_{1-6}$, the electromagnetic dipole operator, $\mathrm{O}_{7}$, and the chromomagnetic dipole operator, $O_{8}$. These operators are evolved from the electroweak scale down to the bottom mass scale using renormalization group equations (RGEs). The partial decay width of the quark-level transition is given by

$$
\Gamma(b \rightarrow s \gamma)=\frac{\alpha G_{F}^{2} m_{b}^{5}}{128 \pi^{4}}\left|V_{t s}^{*} V_{t b} C_{7}\left(\mu_{b}\right)\right|^{2} .
$$

The deviation in the observable is proportional to $\delta C_{7}\left(\mu_{b}\right)$, which can be expressed in terms of the effective Wilson coefficients at the matching scale $\left(\mu_{W}\right)$, given as $\delta C_{7}\left(\mu_{W}\right)$ and $\delta C_{8}\left(\mu_{W}\right)$. The explicit expressions for $C_{7,8}$ can be found in the literature [42]. In 2HDM-like scenarios, such as in the LSS model, an additional contribution comes from the charged Higgs coupling to the quarks, given by

$\mathcal{L}=\frac{\sqrt{2}}{v} H^{+} \bar{t}\left[V_{i j} m_{u i} \lambda_{u}^{i} P_{L} d_{j}+V_{i j} m_{d j} \lambda_{d}^{j} P_{R} d_{j}\right]+$ H.c.

Now, the contribution of the charged Higgs in $\delta C_{7,8}$ can be expressed as $\delta C_{7}(x)$ and $\delta C_{8}(x)$, computed as $[38,43]$

$$
\delta C_{7,8}=\left[\frac{\lambda_{t}^{2}}{3} F_{7,8}^{(1)}(x)-\lambda_{t} \lambda_{b} F_{7,8}^{(2)}(x)\right]
$$

where $x=\frac{\bar{m}_{t}^{2}\left(\mu_{W}\right)}{m_{H^{ \pm}}^{2}}, \lambda_{t} \equiv y_{H^{ \pm} t_{L} b_{R}}$, and $\lambda_{b} \equiv y_{H^{ \pm} t_{R} b_{L}}$, as given in Eq. (17). In the following text, we choose a simpler notation and denote these effective Yukawa couplings as $y_{H t_{L} b_{R}}$ and $y_{H t_{R} b_{L}}$. The detailed expression of the function $F$ is given in Refs. [28,44].

The $B \rightarrow X_{s} \gamma$ branching ratio receives large contributions from the charged Higgs couplings with the top and bottom quarks. We impose the limits on the Wilson coefficient to be in the range $-0.063 \leq \delta C_{7}+0.242$, $\delta C_{8} \leq 0.073$. The theoretical and experimental uncertainties are combined in a quadrature while deriving the limits. It is interesting to note that the relative minus sign between the two contributions (from $W$ and $H^{ \pm}$in the loop) in the general 2HDM model creates destructive interference for some values of the model parameters [28]. However, in the LSS model, the coefficients $\left(\lambda_{t, b}\right)$ depend on the parameters of the strong sector, and both destructive and constructive interference are possible depending on the values of the LSS model parameters. This is true for the other observables as well. Charged Higgs also contributes to $b \rightarrow d \gamma$ decay in the same manner. The coupling of the charged Higgs to the top and down quarks is also similar to Eq. (16), but this coupling is suppressed in this model as $V_{t s} \gg V_{t d}$.

\section{Neutral meson mixing}

In the SM, neutral meson mixing occurs due to the box diagrams with two $W$ exchanges in the loop level. In the case of $B_{d}$ and $B_{s}$ mesons, the hierarchical structure of the CKM matrix and the large mass of the top quark imply that the mixing is dominated by the diagrams involving the top quarks. In 2HDM-like scenarios, the observables related to the neutral-meson mixing receive charged Higgs contributions [45]. Additional diagrams are included by replacing the $W$ lines with charged Higgs ones, yielding the contribution to the mixing as

$$
\Delta m_{q}=\frac{G_{F}^{2}}{24 \pi^{2}}\left(V_{t q} V_{t b}^{*}\right)^{2} \eta_{B} m_{B} m_{t} f_{B q}^{2} I_{\text {tot }}\left(x_{W}, x_{H}, x_{H}\right),
$$

$I_{\mathrm{tot}}=I_{W W}\left(x_{W}\right)+A_{t}^{4} I_{H H}\left(x_{H}, x_{H}\right)+2 A_{t}^{2} I_{W H}\left(x_{W}, x_{H}\right)$,

where $x_{W}=\bar{m}_{t}^{2} / m_{W}^{2}, x_{H}=\bar{m}_{t}^{2} / m_{H}^{2} . I_{W W}, I_{H H}$, and $I_{W H}$ indicate the internal bosonic lines of the corresponding diagrams with an external light quark $q=d, s$. For details of these Inami-Lim functions and other parameters, we refer to Refs. $[28,46]$. We consider the charged Higgs contributions to the $B_{s}^{0}-\bar{B}_{s}^{0}$ mixing only, as the constraints are stronger than $B_{d}^{0}-\bar{B}_{d}^{0}$ mixing [23]. Also, as mentioned earlier, the charged Higgs couplings to the top $(t)$ and down $(d)$ quarks are suppressed in our model. Both experimental measurements and the SM predictions for $B_{s}^{0}-\bar{B}_{s}^{0}$ mixing are given in Table I. We obtain the total contributions to the 
mass splitting from the $W^{ \pm}$bosons, Goldstones, and the charged Higgs boson $\left(H^{ \pm}\right)$through the box graphs. Normalizing $\Delta m_{B_{s}}$ with respect to its SM prediction, we obtain the allowed range at $2 \sigma$ to be $0.675 \leq \frac{\Delta m_{B_{s}}}{\Delta m_{B_{s}}^{\mathrm{SM}}} \leq 1.265$.

\section{3. $Z \rightarrow b \bar{b}$ vertex $\left(R_{b}\right)$}

The $Z \rightarrow b \bar{b}$ vertex has provided opportunities to search for new physics contributions, due to the heavy masses involved in the loop. The radiative corrections at the vertex might imply charged Higgs exchanges in addition to the standard $W$-boson coupling with top and bottom. Precision measurement of the electroweak precision observable $Z \rightarrow b \bar{b}$ branching ratio is measured as

$$
R_{b}=\frac{\Gamma(Z \rightarrow b \bar{b})}{\Gamma(Z \rightarrow \text { hadrons })} .
$$

The modifications in $R_{b}$ due to the charged Higgs contributions at one loop is given by $[30,47]$

$$
\delta R_{b} \simeq-0.7785 \delta g_{\text {new }}^{L} .
$$

Here $\delta g_{\text {new }}^{L}$ is the modification in the $Z b_{L} \bar{b}_{L}$ coupling, calculated from a combination of triangle graphs where $H^{ \pm}$ and the charged Goldstones float inside the loop. We neglect the modification in the $Z b_{R} \bar{b}_{R}$ coupling because it is proportional to $m_{b}^{2}$, compared to $Z b_{L} \bar{b}_{L}$ coupling, which is proportional to $m_{t}^{2}$ [30]. We constrain $\delta R_{b}$ in the $2 \sigma$ range: $-0.00086 \leq \delta R_{b} \leq 0.00182$.

\section{Other flavor observables}

The flavor observables such as $B_{s} \rightarrow \mu \mu$ and $B_{d} \rightarrow \mu \mu$ can also show sizable effects in generic 2HDM. But these observables will have low impact in the LSS model, as $H^{ \pm} l \nu$ coupling is small, resulting in very tiny $\operatorname{BR}\left(H^{ \pm} \rightarrow l \nu\right)$, as discussed in the previous section. In general, standard $2 \mathrm{HDM}$ scenarios require very large values of the $H^{+} l \nu$ couplings with small charged Higgs masses [36] in order to explain the current experimental values (Table I) of $B_{s, d} \rightarrow \mu \mu$. This is very unlikely to happen in the LSS model. The other robust predictions from flavor observables such as $R_{D}, R_{D^{*}}, R_{K}, R_{K^{*}}$, and meson decays depend on BSM scalar couplings $H^{ \pm} l \nu, H^{+} H^{-}(Z / \gamma)$, $\left(H^{0} / h / A^{0}\right) l^{+} l^{-}(l=\mu, \tau)$ in both tree-level and loop-level contributions. These couplings, being small in the LSS scenario, give no effective contribution to the aforementioned flavor observables. Hence, the constraining power of these observables is weaker, and we do not include them in our study.

\section{B. Constraints from flavor physics and $\boldsymbol{R}_{b}$ (Scan A)}

The flavor and electroweak observables depend on the following LSS model parameters:
TABLE II. Values of the parameters and constraints from flavor observables used in the multiparameter Scan A.

\begin{tabular}{lc}
\hline \hline Parameter & Value \\
\hline$y_{i}(i=1 \ldots 5), c, c_{1}, g_{1}, g_{1}^{\prime}$ & $\mathcal{O}(1)$ \\
$y_{1 b}, y_{2 b}$ & $\leq 0.10$ \\
$f$ & $\leq 2 \mathrm{TeV}$ \\
$M$ & $\leq 3 \mathrm{TeV}$ \\
VEV & $246 \mathrm{GeV}$ \\
$\delta C_{7}+0.242 \delta C_{8}$ & $(-0.063,0.073)$ \\
$\Delta m_{B_{s}}$ & $(0.675,1.265)$ \\
\hline$m_{B_{s}}^{\mathrm{SM}}$ & $(-0.00086,0.00182)$ \\
$\delta R_{b}$ & \\
\hline \hline
\end{tabular}

$$
y_{1}, y_{2}, y_{3}, y_{4}, y_{5}, c, c^{\prime}, g_{1}, g_{1}^{\prime}, y_{1 b}, y_{2 b}, f \text {, and } M \text {. }
$$

The values of the top-sector Yukawa couplings $y_{i}, i=$ $1,2 \ldots 5$ are taken to be of the order of unity, which is a natural choice, as they, as a combination, provide the topquark mass with VEVs $v_{1}, v_{2} \sim 100 \mathrm{GeV}$. Three cases of the LSS model (Cases I, II, and III) have different bottom Yukawa structures, depending on the values of the Yukawa couplings $y_{1 b}, y_{2 b}$. The Yukawa couplings in the bottom sector, $y_{1 b}$ and $y_{2 b}$, are responsible for the bottom-quark mass. To generate the bottom mass, which is 2 orders of magnitude smaller than the top mass, $y_{1 b}$ and $y_{2 b}$ are varied in an optimum range, much smaller than the other Yukawa couplings. $y_{1 b}$ and $y_{2 b}$ are varied over a range $\left|y_{i b}\right| \leq 0.10$. The intermediate Yukawa couplings $y_{00}^{-}, y_{10}^{-}$have combinations of the form $y_{1 b} \cos \beta-y_{2 b} \sin \beta$, which chart out relative contributions of the $y_{1 b}, y_{2 b}$. The rest of the parameters $-c, c_{1}, g_{1}$, and $g_{1}^{\prime}$ - are considered to be $\mathcal{O}(1)$.

We have used the relation $M=1.5 \times f$, where $f$ takes values up to $2 \mathrm{TeV}$, and we have fixed the VEV at $246 \mathrm{GeV}$. Hence, we perform a random multiparameter scan in 12 parameter space. As noted in Ref. [21], to prevent the VEVs from running away to $\infty$, sufficient conditions are imposed, given as $m_{1,2}^{2}>0$ and $\left(m_{1}^{2} m_{2}^{2}-b^{4}\right)<0$, where $b^{2}$ is real. We have implemented these criteria in the multiparameter scan as well. As the flavor constraints are expected to be overwhelmed by the LHC constraints, we work in a more relaxed framework in terms of LHC parameters in order to emphasize the flavor intricacies of this type of setup. The top and Higgs masses are fixed in the scan within a liberal window of $20 \mathrm{GeV}$ and $10 \mathrm{GeV}$, respectively. ${ }^{5}$ In order to see the effects emanating from the flavor sector, the flavor observables are kept within their current allowed limits, as listed in Table I. Along with that, the bottom mass is also varied within $3-5 \mathrm{GeV}$. We list the values of the parameters and constraints in the first scan, Scan A, in Table II.

\footnotetext{
${ }^{5}$ Note that we impose a much stronger constraint on these parameters in the next scan, Scan B.
} 

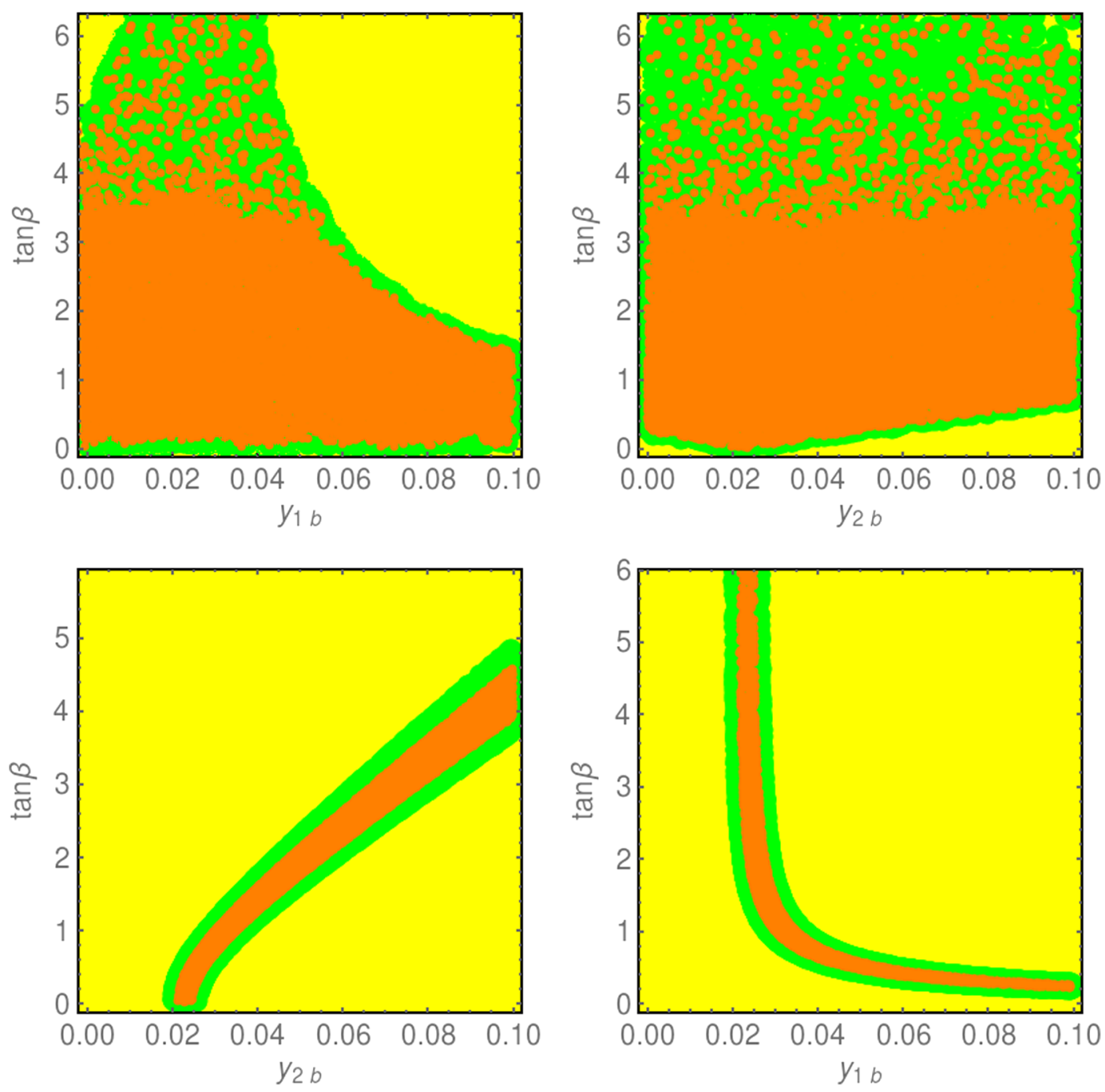

FIG. 1. Scan $\mathbf{A}$-Variation of $\tan \beta$ as a function of $y_{1 b}$ and $y_{2 b}$ in three cases: Case I (upper panel), Case II (lower panel left), and Case III (lower panel right), after imposing constraints from $R_{b}$ and $B_{s}-\bar{B}_{s}$ combined (green) and $B \rightarrow X_{s} \gamma$ (red).

In the following, we present Figs. 1 and 2 for three different cases, as specified earlier. In Fig. 1, we plot Case I in the top panel and Cases II (left) and III (right) in the bottom panel. In Fig. 2, we plot Cases I, II, and III in the first, second, and third columns, respectively. In these figures, the yellow points are generated by the model parameter space that is allowed after the imposition of constraints on them, as given in the top part of Table II. With further imposition of constraints from the flavor and EW observables, green and red points depict the projections of the allowed parameter regions that satisfy the constraints (Table II) from $R_{b}$ and $B_{s}-\bar{B}_{s}$ mixing together and $B \rightarrow X_{s} \gamma$, respectively. It is found that $R_{b}$ and $B_{s}-\bar{B}_{s}$ satisfy almost the same parameter space. Hence, we choose to impose combined constraints in the plots. The flavor bound from $B \rightarrow X_{s} \gamma$ decay is found to be the most constraining for the LSS model. Hence, in the following discussion about the model parameter space constrained from flavor observables, we shall refer mainly to the strongest constraint that comes from $B \rightarrow X_{s} \gamma$.
We show the variation of the Yukawa parameters with $\tan \beta$ in Figs. 1 and 2. In this model, both $m_{1}^{2}$ and $m_{2}^{2}$ get contributions of the same order of magnitude, through some combinations of the Yukawa couplings, $y_{i}$, $i=1,2 \ldots .5$, which are of the same order. Hence, $\tan \beta$ in the LSS model, being written in terms of the ratio of $m_{1}^{2}$ and $m_{2}^{2}$, is expected to lie in the vicinity of unity. It is reflected from the plots that overall small $\tan \beta$ values are preferred. As $\tan \beta$ values gradually increase from $<1$ to $>1$, up to $\tan \beta \sim 5$, the relative dominances of the $\sin \beta$ - and $\cos \beta$ dependent terms in Eqs. (16) and (17) increase and decrease, respectively. In Fig. 1, in the Case I scenario, where both $y_{i b} \neq 0$, flavor constraints seem to rule out the higher $\tan \beta$ region for higher $y_{1 b}$ values along with the lower $\tan \beta$ region for higher $y_{2 b}$ values. This happens as the contributions to $y_{1 b} \cos \beta-y_{2 b} \sin \beta$ do not exactly cancel each other, thus paving the way for increased contribution to the observables ( $B \rightarrow X_{s} \gamma$, etc.), constrained by experimental bounds. 

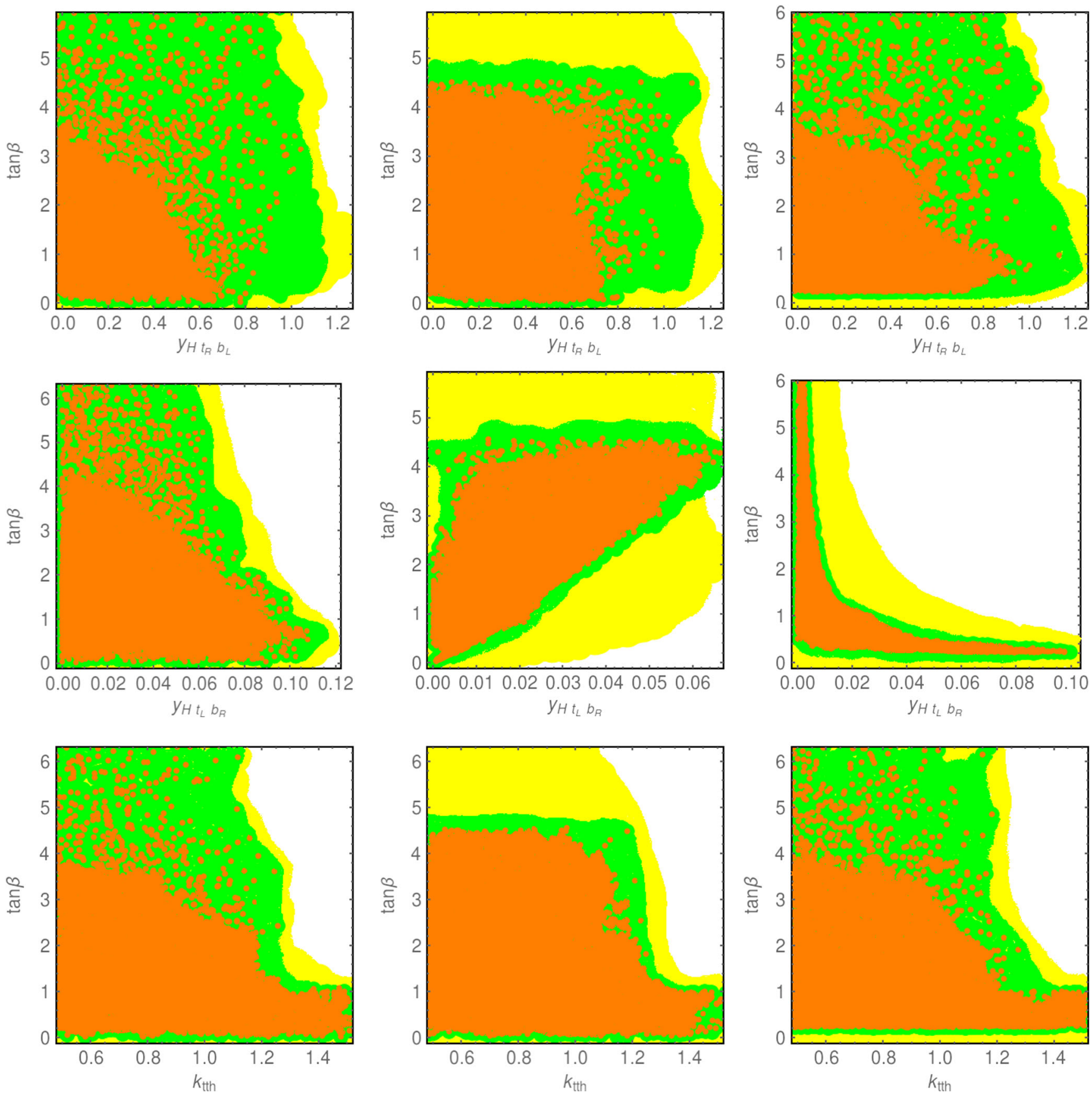

FIG. 2. Scan A-Variation of $\tan \beta$ with the effective Yukawa couplings and $k_{t t h}$ in three cases: Case I (left column), Case II (middle column), and Case III (right column), after imposing constraints from $R_{b}$ and $B_{s}-\bar{B}_{s}$ combined (green) and $B \rightarrow X_{s} \gamma$ (red).

From Fig. 1, it is clear that the maximum value of $\tan \beta$ can be 5.0 for Case II, whereas for Case III, $\tan \beta$ up to 6 is mostly preferred by the flavor constraints. ${ }^{6}$ In Case II, for $y_{1 b}=0$, the $\lambda_{t}$ values in Eq. (27) remain significant only for larger $y_{2 b}$ and $\sin \beta$, which is related to $\tan \beta$. Only with

\footnotetext{
${ }^{6}$ We do not show the region for $\tan \beta \geq 6$, as this region is less favored by the LSS model, as well as the flavor observables, resulting in very few points in the plot.
}

large $y_{2 b}$ and $\sin \beta$ does $\lambda_{t}$ become larger, which is paramount to fitting the flavor data. Similarly, for Case III with $y_{2 b}=0$, after a certain $y_{1 b}(\geq 0.02)$, smaller values of $\tan \beta$ are preferred with increasing $y_{1 b}$, as $\cos \beta$ becomes significant in that range and fails to provide the required values of $\lambda_{t}$ to satisfy the flavor constraints. Moreover, in Case III, $\tan \beta$ cannot obtain a very small value; the $\tan \beta<0.5$ region is disfavored from the flavor observables. Therefore, Case II with $y_{1 b}=0$ differs 
significantly from Case I with all Yukawas nonzero, whereas Case III with $y_{2 b}=0$ remains relatively unaffected.

The effective Yukawa pattern subsequently reveals the dominance of either $y_{1 b}$ or $y_{2 b}$ through their contributions in Eq. (17). From Fig. 2, we can see different ranges of $y_{H t_{R} b_{L}}$ and $y_{H t_{L} b_{R}}$. The allowed $y_{H t_{R} b_{L}}$ values are 1 order of magnitude higher than $y_{H t_{L} b_{R}}$ values, up to $\mathcal{O}(1.0)$, as opposed to $\mathcal{O}\left(10^{-1}\right)$. This is due to the fact that $y_{H t_{R} b_{L}}$ and $y_{H t_{L} b_{R}}$ are primarily dominated by the top- and bottomsector Yukawa couplings $\left(y_{i}, i=1,2\right.$ vs $\left.y_{i b}, i=1,2\right)$, respectively. They are also functions of the left and right top-sector mixing elements in $R_{L}$ and $R_{R}$, which are of similar orders of magnitude, being functions of $y_{i}$ 's $(i=1,2 \ldots 5)$. The effective Yukawa coupling $y_{H t_{R} b_{L}}$ is a combination of top-sector Yukawas, and there is no direct $y_{1 b}, y_{2 b}$ contribution. Hence, $y_{H t_{R} b_{L}}$ almost produces similar allowed regions after flavor constraints in three different cases. This is reflected in the top row of Fig. 2, where we plot $y_{H t_{R} b_{L}}$ vs $\tan \beta$. The flavor-allowed regions in Case III are somewhat similar to those of Case I, but Case II stands apart, where relatively larger values of $\tan \beta(>4.5)$ are not allowed.

The middle row of Fig. 2 shows the allowed regions of the effective Yukawa coupling $y_{H t_{L} b_{R}}$ with $\tan \beta$ for three cases. Large values of $y_{H_{L} b_{R}}(\sim 0.1)$ are allowed for $\tan \beta<1.5$ in Case I. Larger values of $\tan \beta(\sim 4-5)$ are favored in the region where $y_{H t_{L} b_{R}}$ is $>0.02$ in Case II; whereas in Case III, $\tan \beta \leq 1$ is favored. In Case III, smaller values of $y_{1 b}(<0.02)$ prefer a large $\tan \beta$. Another interesting feature of Case III is that a very small value of $\tan \beta(<0.2)$ is disfavored by $B \rightarrow X_{s} \gamma$, as shown in all the plots in the right column of Fig. 2.

We also plot the $\tan \beta$ dependence of $k_{t t h}$ in Fig. 2. It is found that, in the experimentally allowed window of $0.7<k_{t t h}<1.4, \tan \beta$ is strongly preferred within the range $0.2-4.0$ in Cases I and III, but values of $\tan \beta$ larger than 4 are also allowed by $B \rightarrow X_{s} \gamma$. In Case II, the upper limit on $\tan \beta$ is 4.0. The allowed values of the charged Higgs mass are almost the same in these three cases. In the next section, we discuss the LHC constraints, starting from combined flavor constraints on the charged Higgs mass.

\section{Constraints from the LHC (Scan B)}

At the LHC, properties of the Higgs boson are measured in several channels [48], which indicates that the couplings of the $125 \mathrm{GeV}$ Higgs to the gauge bosons $(h V V)$ and to the fermions $(h f \bar{f})$ are consistent with the SM prediction [20,49-51], even though some mismatches are still there in the fermionic coupling measurements. These measurements are found to be in favor of the alignment limit $[52,53]$ in a 2 HDM-like setup i.e., $|\sin (\beta-\alpha)| \sim 1$. The nonobservation of anomalous nature of the $h V V$ coupling strongly indicates that any BSM contribution is suppressed in the Higgs-gauge boson interaction, implying $|\cos (\beta-\alpha)| \sim 0[54,55]$. Hence, the $h V V$ coupling in the LSS model, which exhibits a 2HDM-like structure, is suppressed. Among the fermionic couplings, we only constrain the Higgs coupling to the third generation of quarks. We allow both positive and negative $h V V$ and $h t \bar{t}$ couplings leading to both constructive and destructive interference in the Higgs observables. ATLAS and CMS experiments have imposed bounds on the couplings of the SM Higgs [48] by looking at different production and decay modes of the Higgs boson. The current limit on htt coupling is dependent on different assumptions held during the statistical fit, as shown in Ref. [48]. After inspecting the limits carefully, we perform our search in the window of $0.70<k_{t t h}<1.4$, where $k_{t t h}$ is defined as the ratio of the measured $h t t$ coupling compared to its $\mathrm{SM}$ value [see Eq. (12)]. In the alignment limit, the $h b \bar{b}$ coupling is $\sim m_{b} / v$, which is maintained throughout the multiparameter scan.

We derive the masses of the SM and BSM particles in terms of the model parameters. We calculate the top mass in the $\overline{\mathrm{MS}}$ scheme [56,57] and constrain it in the range $156-170 \mathrm{GeV}$ around $m_{t}(\overline{\mathrm{MS}})=163 \mathrm{GeV}$. The Higgs mass $[58,59]$ is considered in the range $123-127 \mathrm{GeV}$. The LEP experiment excludes the charged Higgs masses below $80 \mathrm{GeV}$ [60]. At the LHC, searches for the charged Higgs have been performed through various decay channels, $H^{ \pm} \rightarrow c s$ [61,62], $t b$ [63,64], and $\nu \tau^{ \pm}$[65], and most of these searches exclude $m_{H}^{ \pm}<m_{t}$. However, the experimental searches [66] in the $H^{ \pm} \rightarrow t b$ channel are favored at higher masses of $H^{ \pm}$, as predicted in other BSM models-for example, in supersymmetric scenarios [67]. Incidentally, also in the LSS model we get $\operatorname{BR}\left(H^{ \pm} \rightarrow\right.$ $t b \sim 1$ ), satisfying a large parameter space of the model. Electroweak precision measurements [34,39] require the charged Higgs mass to be close to the mass of one the neutral Higgses, which is also satisfied in our model (also shown in Ref. [22]). Different searches at the LHC have imposed limits on the masses of the vector like quarks as well, and typically they are within the range of 1 to $1.4 \mathrm{TeV}$ [32]. We list the values of all the constraining observables of the second Scan B in Table III. The model parameters follow the same values as given in Table II.

In Fig. 3, the magenta region shows the model parameter space allowed by combination of the flavor constraints and the $R_{b}$ (allowed points of Scan $\mathbf{A}$ ). We constrain the magenta region further with observables of the Scan $\mathbf{B}$ as given in Table III. The result of Scan $\mathbf{B}$ is shown by the blue points. The first row of Fig. 3 shows the allowed parameter space of the Yukawa couplings, which contributes to the couplings of charged Higgs to the third generation quarks. Interestingly, after both the scans, we get very distinct patterns for the three cases. Parameter space that satisfies all the constraints (blue points) is uniformly distributed in Case I. In Case II, negative values of $y_{H t_{R} b_{L}}$ are more 
TABLE III. The experimental constraints used in the multiparameter Scan B.

\begin{tabular}{lc}
\hline \hline Quantity & Constraints \\
\hline$|\sin (\beta-\alpha)|$ & $\sim 1$ \\
$k_{t t h}$ & $0.7-1.4$ \\
$m_{t}$ & $(156,170) \mathrm{GeV}$ \\
$m_{h}$ & $(123,127) \mathrm{GeV}$ \\
$m_{b}$ & $(3,5) \mathrm{GeV}$ \\
$m_{H}^{ \pm}$ & $>m_{t}$ \\
$m_{B}, m_{T}$ & $>1.4 \mathrm{TeV}$ \\
\hline \hline
\end{tabular}

preferred, and in Case III, very small values of $y_{H t_{L} b_{R}}$ are not allowed after the imposition of LHC constraints.

In the second row of Fig. 3, we show the plot of $\tan \beta$ vs the mass of the charged Higgs boson. Mass of the charged Higgs is a function of $\tan \beta, \lambda_{5}^{\prime}$, and $b^{2}$, which depend intrinsically on other model parameters. The most important constraint form the LHC is on the htt coupling, which again depends on the same set of model parameters. The parameter space that survives after Scan B, predicts such ranges of $m_{H}^{ \pm}$as shown by blue points in Fig 3. The preferred values of charged Higgs mass stay well above
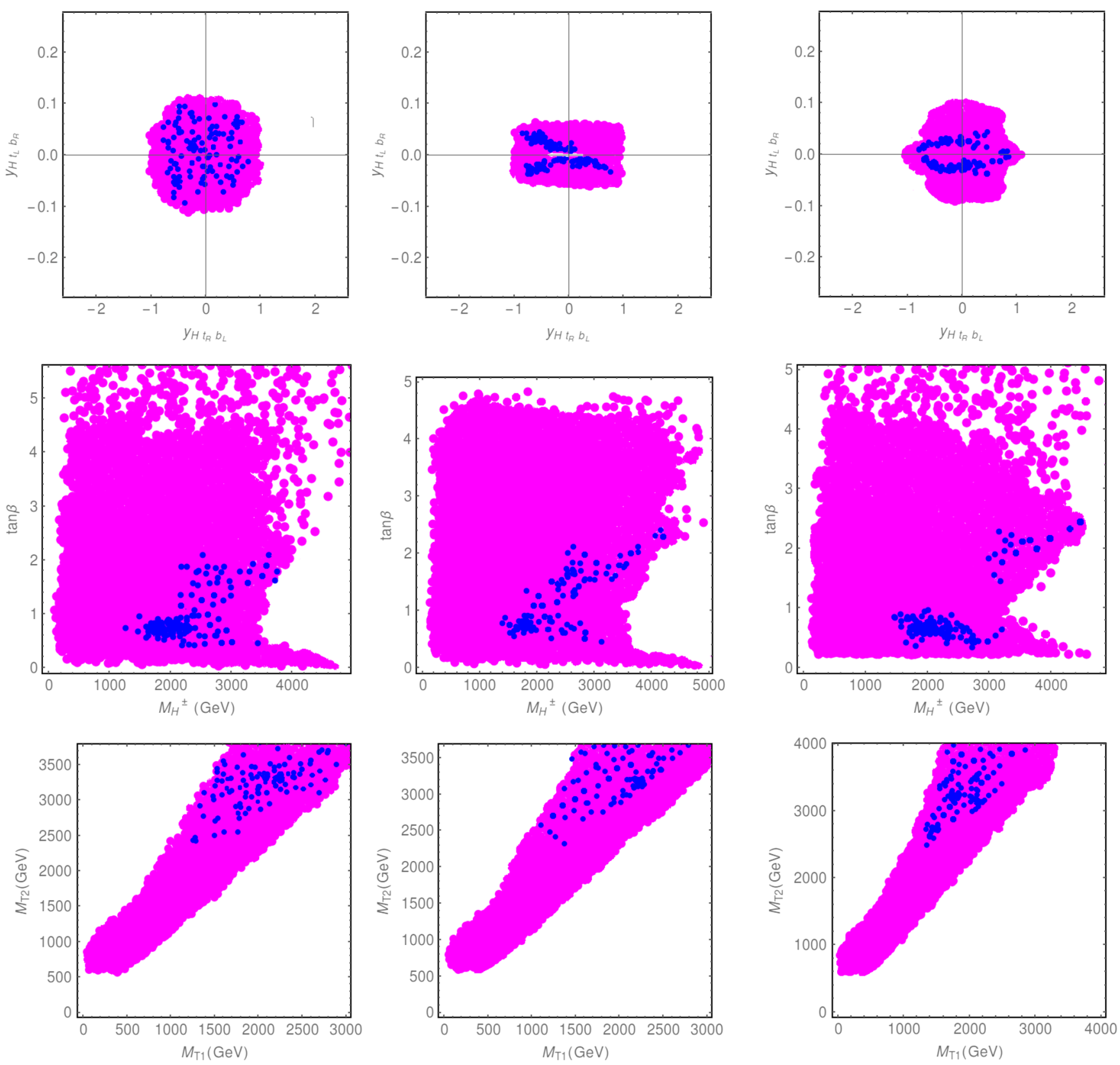

FIG. 3. Scan B-LSS model parameter space after imposing constraints from flavor observables and $R_{b}$ (magenta) and further imposition of LHC observables (blue) in three cases: Case I (left column), Case II (middle column), and Case III (right column). 
$1 \mathrm{TeV}$, the smallest allowed value being $\sim 1.3 \mathrm{TeV}$, from Scan B. Case I shows more preference towards smaller values of $m_{H}^{ \pm}$compared to Case II. Overall, the plots in the first and second row reflect mainly the correlation among the flavor physics observables, $R_{b}$ and the Higgs Yukawa measurements at the LHC, in the allowed LSS model parameter space.

The bottom row confirms that the toplike VLQ partners are well above $1.3 \mathrm{TeV}$ after Scan $\mathbf{B}$, and the allowed region is almost the same in the three cases. Our results also predict that a similar inference is true for the bottomlike VLQs. In general, we find that the limits on VLQ masses and the charged Higgs mass are more stringent after the imposition of the LHC bounds-i.e, Scan B-specifically due to the alignment condition. A similar conclusion can be made for the extra neutral and charged gauge bosons, such as $B^{\prime}$ and $W^{\prime}$, where the allowed masses are pushed to even higher values, $\geq 2.2 \mathrm{TeV}$. This also takes care of the constraints coming from electroweak precision tests (EWPT) [22].

\section{CONCLUSION}

We reintroduce the LSS little Higgs model that acts as an UV-complete model to produce an effective $2 \mathrm{HDM}$ at the EW scale, along with other BSM species, such as vectorlike fermions, extra gauge bosons, and scalars. Compared to the generic 2HDM, emergent 2HDM from the LSS model will be predictive in nature, as the Yukawa structure here will be dictated by a bigger symmetry. In this work, emergent 2HDM from the LSS model is discussed from the viewpoint of standard 2HDM to determine the crucial phenomenological differences. We bring out both flavor and LHC constraints together from the arsenal, ably aided by EW precision observable $R_{b}$ to pin down the allowed parameter space of the LSS model. In the context of an emergent $2 \mathrm{HDM}$, the qualitative features of an $S U(6) / S p(6)$ composite Higgs model are expected to be similar to those for the LSS model, albeit with obvious differences in parametric dependences.

In the flavor sector, variants of models are constructed from the bottom Yukawa perspective, and we have found that the case with both $y_{i b} \neq 0$ is relatively less constrained compared to the cases with either of them set to zero. We deploy different flavor observables, potentially important to the charged Higgs sector. Among them, the $B \rightarrow X_{s} \gamma$ branching ratio is found to be the most constraining, while $B_{s}-\bar{B}_{s}$ mixing and EWPT observable $R_{b}$ constraints are relatively liberate, though they are not widely off. While the flavor constraints keep the charged Higgs fairly relaxed, the $\tan \beta$ gets more restricted $(\leq 5)$ compared to the usual 2HDM. The effective charged Higgs Yukawas, $y_{H t_{L} b_{R}}$ and $y_{H t_{R} b_{L}}$, show very distinct $\tan \beta$ dependences in different cases (Cases I, II, and III), manifesting different flavor patterns.

With respect to the combined flavor and LHC constraints, charged Higgs mass $\left(m_{H}^{ \pm}\right)$and $\tan \beta$ are bound more tightly in the emergent 2HDM from the LSS model than in the usual 2HDM. Even when some of the constrained 2HDM scenarios rule out the charged Higgs only in the sub-TeV region, here in the LSS model, combined flavor and LHC bounds push the charged Higgs mass lower bound to $1.3 \mathrm{TeV}$. Similarly, $\tan \beta$ is spread over a wider range in general $2 \mathrm{HDM}$ as opposed to a narrow range of $(0.5-3)$ in the different LSS scenarios. This is a reflection of the predictive nature of the Yukawa sector in the LSS model, where the Higgs mass, top mass and top Yukawa couplings are fixed in terms of strong sector parameters of the LSS model; whereas these quantities were easily arranged in the construction of the general 2HDM through the enforcement of a $Z_{2}$ symmetry.

These types of LH models with a large number of parameters are often very fine-tuned, as multiple parameters contribute to a single observable, such as the Higgs mass, which is very precisely known. It is worthwhile to know in the future how the three different flavor scenarios, as discussed above, can have an impact on the charged Higgs phenomenology. In general, we find that the charged Higgs, along with other neutral BSM particles, is placed at a mass well above $1 \mathrm{TeV}$. Hence, their decay products are expected to be highly energetic, such that one can use them to improvise different LHC search techniques. In one of the earlier LHC searches [68], a heavy charged Higgs similar to this scenario, which dominantly decays through the $t b$ mode, was probed in all jet final states, using boosted properties of top and $W$. These kinds of searches will have better prospects at the advanced run of the LHC with enhanced luminosity and energy.

\section{ACKNOWLEDGMENTS}

S. S. thanks UGC for the DS Kothari postdoctoral fellowship Grant No. F.4-2/2006 (BSR)/PH/17-18/0126. N. K. acknowledges the support from the Dr. D.S. Kothari Postdoctoral scheme (No. 201819-PH/18-19/ 0013). S. S. thanks Dr. Shrihari Gopalakrishna for his guidance as Ph.D. supervisor to study this model. S. S. also thanks Dr. Shrihari Gopalakrishna and Dr. Tuhinsubhra Mukherjee for their inputs on the LSS model construction and phenomenology during the course of an earlier work [22]. We also sincerely thank the referee for a number of important, thought-provoking suggestions that have immensely helped us to improve our manuscript. 
[1] E. Gildener, Gauge-symmetry hierarchies, Phys. Rev. D 14, 1667 (1976).

[2] P. Fayet, Supersymmetry and weak, electromagnetic and strong interactions, Phys. Lett. 64B, 159 (1976).

[3] N. Arkani-Hamed, A. G. Cohen, and H. Georgi, Electroweak symmetry breaking from dimensional deconstruction, Phys. Lett. B 513, 232 (2001).

[4] D. B. Kaplan and H. Georgi, $S U(2) \times U(1)$ Breaking by vacuum misalignment, Phys. Lett. 136B, 183 (1984).

[5] H.-C. Cheng and I. Low, Little hierarchy, little Higgses, and a little symmetry, J. High Energy Phys. 08 (2004) 061.

[6] H.-C. Cheng and I. Low, TeV symmetry and the little hierarchy problem, J. High Energy Phys. 09 (2003) 051.

[7] I. Low, $T$ parity and the littlest Higgs, J. High Energy Phys. 10 (2004) 067.

[8] D. E. Kaplan and M. Schmaltz, The little Higgs from a simple group, J. High Energy Phys. 10 (2003) 039.

[9] R. Contino, The Higgs as a composite Nambu-Goldstone boson, in Theoretical Advanced Study Institute in Elementary Particle Physics: Physics of the Large and the Small (2011), pp. 235-306, https://doi.org/10.1142/ 9789814327183_0005.

[10] K. Agashe, R. Contino, and A. Pomarol, The minimal composite Higgs model, Nucl. Phys. B719, 165 (2005).

[11] G. Panico, M. Redi, A. Tesi, and A. Wulzer, On the tuning and the mass of the composite Higgs, J. High Energy Phys. 03 (2013) 051.

[12] J. Berger, J. Hubisz, and M. Perelstein, A fermionic top partner: Naturalness and the LHC, J. High Energy Phys. 07 (2012) 016.

[13] F. Sannino, A. Strumia, A. Tesi, and E. Vigiani, Fundamental partial compositeness, J. High Energy Phys. 11 (2016) 029.

[14] M. Schmaltz and D. Tucker-Smith, Little Higgs review, Annu. Rev. Nucl. Part. Sci. 55, 229 (2005).

[15] M. Perelstein, Little Higgs models and their phenomenology, Prog. Part. Nucl. Phys. 58, 247 (2007).

[16] T. Ma and G. Cacciapaglia, Fundamental Composite 2HDM: $S U(N)$ with 4 flavours, J. High Energy Phys. 03 (2016) 211.

[17] C. Cai, G. Cacciapaglia, and H.-H. Zhang, Vacuum alignment in a composite 2HDM, J. High Energy Phys. 01 (2019) 130 .

[18] E. Bertuzzo, T. S. Ray, H. de Sandes, and C. A. Savoy, On Composite Two Higgs Doublet Models, J. High Energy Phys. 05 (2013) 153.

[19] J. Mrazek, A. Pomarol, R. Rattazzi, M. Redi, J. Serra, and A. Wulzer, The other natural two Higgs doublet model, Nucl. Phys. B853, 1 (2011).

[20] G. Branco, P. Ferreira, L. Lavoura, M. Rebelo, M. Sher, and J. P. Silva, Theory and phenomenology of two-Higgsdoublet models, Phys. Rep. 516, 1 (2012).

[21] I. Low, W. Skiba, and D. Tucker-Smith, Little Higgses from an antisymmetric condensate, Phys. Rev. D 66, 072001 (2002).

[22] S. Gopalakrishna, T. S. Mukherjee, and S. Sadhukhan, Status and prospects of the two-Higgs-doublet $S U(6) / S p(6)$ littleHiggs model and the alignment limit, Phys. Rev. D 94, 015034 (2016).
[23] Y. Amhis et al. (HFLAV Collaboration), Averages of $b$ hadron, $c$-hadron, and $\tau$-lepton properties as of summer 2016, Eur. Phys. J. C 77, 895 (2017).

[24] F. del Aguila, L. Ametller, J. I. Illana, J. Santiago, P. Talavera, and R. Vega-Morales, The full lepton flavor of the littlest Higgs model with $T$-parity, J. High Energy Phys. 07 (2019) 154.

[25] S. Fajfer and J. F. Kamenik, On the flavor structure of the littlest Higgs model, J. High Energy Phys. 12 (2007) 074.

[26] I. I. Bigi, M. Blanke, A. J. Buras, and S. Recksiegel, CP violation in D0-anti-D0 oscillations: General considerations and applications to the littlest Higgs model with $T$-parity, J. High Energy Phys. 07 (2009) 097.

[27] T. Han, H.E. Logan, B. McElrath, and L.-T. Wang, Phenomenology of the little Higgs model, Phys. Rev. D 67, 095004 (2003).

[28] F. Mahmoudi and O. Stal, Flavor constraints on the twoHiggs-doublet model with general Yukawa couplings, Phys. Rev. D 81, 035016 (2010).

[29] V. Barger, J. L. Hewett, and R. J. N. Phillips, New constraints on the charged Higgs sector in two-Higgs-doublet models, Phys. Rev. D 41, 3421 (1990).

[30] H. E. Haber and H. E. Logan, Radiative corrections to the $Z b \bar{b}$ vertex and constraints on extended Higgs sectors, Phys. Rev. D 62, 015011 (2000).

[31] A. Banerjee, G. Bhattacharyya, and N. Kumar, Impact of Yukawa-like dimension-five operators on the GeorgiMachacek model, Phys. Rev. D 99, 035028 (2019).

[32] A. Buckley, J. Butterworth, L. Corpe, D. Huang, and P. Sun, New sensitivity of current LHC measurements to vector-like quarks, SciPost Phys. 9, 069 (2020).

[33] S. Rappoccio, The experimental status of direct searches for exotic physics beyond the Standard Model at the Large Hadron Collider, Rev. Phys. 4, 100027 (2019).

[34] S. Schael et al. (ALEPH, DELPHI, L3, OPAL, SLD, LEP Electroweak Working Group, SLD Electroweak Group, SLD Heavy Flavour Group Collaboration), Precision electroweak measurements on the $Z$ resonance, Phys. Rep. 427, 257 (2006).

[35] S. Gopalakrishna, T. S. Mukherjee, and S. Sadhukhan, Extra neutral scalars with vectorlike fermions at the LHC, Phys. Rev. D 93, 055004 (2016).

[36] A. Crivellin, A. Kokulu, and C. Greub, Flavorphenomenology of two-Higgs-doublet models with generic Yukawa structure, Phys. Rev. D 87, 094031 (2013).

[37] K. Ishiwata, Z. Ligeti, and M. B. Wise, New vector-like fermions and flavor physics, J. High Energy Phys. 10 (2015) 027.

[38] M. Misiak et al., Updated NNLO QCD Predictions for the Weak Radiative $b$-Meson Decays, Phys. Rev. Lett. 114, 221801 (2015).

[39] J. Haller, A. Hoecker, R. Kogler, K. Mnig, T. Peiffer, and J. Stelzer, Update of the global electroweak fit and constraints on two-Higgs-doublet models, Eur. Phys. J. C 78, 675 (2018).

[40] C. Bobeth, M. Gorbahn, T. Hermann, M. Misiak, E. Stamou, and M. Steinhauser, $B_{s, d} \rightarrow l^{+} l^{-}$in the Standard Model with Reduced Theoretical Uncertainty, Phys. Rev. Lett. 112, 101801 (2014). 
[41] R. Aaij et al. (LHCb Collaboration), Measurement of the $B_{s}^{0} \rightarrow \mu^{+} \mu^{-}$Branching Fraction and Effective Lifetime and Search for $B^{0} \rightarrow \mu^{+} \mu^{-}$Decays, Phys. Rev. Lett. 118, 191801 (2017).

[42] G. Buchalla, A. J. Buras, and M. E. Lautenbacher, Weak decays beyond leading logarithms, Rev. Mod. Phys. 68, 1125 (1996).

[43] Q.-Y. Hu, X.-Q. Li, and Y.-D. Yang, $B^{0} \rightarrow K^{* 0} \mu^{+} \mu^{-}$decay in the aligned two-Higgs-doublet model, Eur. Phys. J. C 77, 190 (2017).

[44] M. Ciuchini, G. Degrassi, P. Gambino, and G. F. Giudice, Next-to-leading QCD corrections to $B \rightarrow X_{s} \gamma$ : Standard model and two Higgs doublet model, Nucl. Phys. B527, 21 (1998).

[45] A. W. El Kaffas, P. Osland, and O. M. Ogreid, Constraining the two-Higgs-doublet-model parameter space, Phys. Rev. D 76, 095001 (2007).

[46] L. F. Abbott, P. Sikivie, and M. B. Wise, Constraints on charged Higgs couplings, Phys. Rev. D 21, 1393 (1980).

[47] H. E. Logan, Ph.D. thesis, University of California, Santa Cruz, 1999, arXiv:hep-ph/9906332.

[48] G. Aad et al. (ATLAS Collaboration), Combined measurements of Higgs boson production and decay using up to $80 \mathrm{fb}^{-1}$ of proton-proton collision data at $\sqrt{s}=13 \mathrm{TeV}$ collected with the ATLAS experiment, Phys. Rev. D 101, 012002 (2020).

[49] N. Chen, T. Han, S. Su, W. Su, and Y. Wu, Type-II 2HDM under the precision measurements at the $Z$-pole and a Higgs factory, J. High Energy Phys. 03 (2019) 023.

[50] N. Chen, T. Han, S. Li, S. Su, W. Su, and Y. Wu, Type-I 2HDM under the Higgs and electroweak precision measurements, J. High Energy Phys. 08 (2020) 131.

[51] N. Chen, T. Han, S. Su, W. Su, and Y. Wu, Implication of Higgs precision measurement on new physics, in International Workshop on Future Linear Colliders, 2019, arXiv:1901.09067.

[52] G. Bhattacharyya and D. Das, Scalar sector of two-Higgsdoublet models: A minireview, Pramana 87, 40 (2016).

[53] A. Banerjee and G. Bhattacharyya, Probing the Higgs boson through Yukawa force, Nucl. Phys. B961, 115261 (2020).

[54] N. Chen, J. Gu, T. Han, H. Li, Z. Liu, H. Song, S. Su, W. Su, Y. Wu, and J. M. Yang, New physics implication of Higgs precision measurements, Int. J. Mod. Phys. A 34, 1940012 (2019).

[55] W. Su, Probing loop effects in wrong-sign Yukawa region of 2HDM, arXiv:1910.06269.
[56] S. Alekhin, A. Djouadi, and S. Moch, The top quark and Higgs boson masses and the stability of the electroweak vacuum, Phys. Lett. B 716, 214 (2012).

[57] M. Tanabashi et al. (Particle Data Group Collaboration), Review of particle physics, Phys. Rev. D 98, 030001 (2018).

[58] G. Aad et al. (ATLAS Collaboration), Observation of a new particle in the search for the Standard Model Higgs boson with the ATLAS detector at the LHC, Phys. Lett. B 716, 1 (2012).

[59] S. Chatrchyan et al. (CMS Collaboration), Observation of a new boson at a mass of $125 \mathrm{GeV}$ with the CMS Experiment at the LHC, Phys. Lett. B 716, 30 (2012).

[60] G. Abbiendi et al. (ALEPH, DELPHI, L3, OPAL, and LEP Collaborations), Search for charged Higgs bosons: Combined results using LEP data, Eur. Phys. J. C 73, 2463 (2013).

[61] G. Aad et al. (ATLAS Collaboration), Search for a light charged Higgs boson in the decay channel $H^{+} \rightarrow c \bar{s}$ in $t \bar{t}$ events using pp collisions at $\sqrt{s}=7 \mathrm{TeV}$ with the ATLAS detector, Eur. Phys. J. C 73, 2465 (2013).

[62] CMS Collaboration, Search for a light charged Higgs boson in the $H^{ \pm} \rightarrow c s$ channel at $13 \mathrm{TeV}$, Technical Report No. CMS-PAS-HIG-18-021, CERN, Geneva, 2019.

[63] M. Aaboud et al. (ATLAS Collaboration), Search for charged Higgs bosons decaying into top and bottom quarks at $\sqrt{s}=13 \mathrm{TeV}$ with the ATLAS detector, J. High Energy Phys. 11 (2018) 085.

[64] A. M. Sirunyan et al. (CMS Collaboration), Search for a charged Higgs boson decaying into top and bottom quarks in events with electrons or muons in proton-proton collisions at $\sqrt{\mathrm{s}}=13 \mathrm{TeV}$, J. High Energy Phys. 01 (2020) 096.

[65] A. M. Sirunyan et al. (CMS Collaboration), Search for charged Higgs bosons in the $H^{ \pm} \rightarrow \tau^{ \pm} \nu_{\tau}$ decay channel in proton-proton collisions at $\sqrt{s}=13 \mathrm{TeV}$, J. High Energy Phys. 07 (2019) 142.

[66] D. de Florian et al. (LHC Higgs Cross Section Working Group Collaboration), Handbook of LHC Higgs cross sections: 4. Deciphering the nature of the Higgs sector, arXiv:1610.07922.

[67] Y. Hiçyılmaz, L. Selbuz, L. Solmaz, and C. S. Ün, Charged Higgs boson in MSSM and beyond, Phys. Rev. D 97, 115041 (2018).

[68] A. M. Sirunyan et al. (CMS Collaboration), Search for charged Higgs bosons decaying into a top and a bottom quark in the all-jet final state of $p p$ collisions at $\sqrt{s}=13 \mathrm{TeV}$, J. High Energy Phys. 07 (2020) 126. 